Algebraic 8 Geometric $\mathcal{T}$ opology

Volume 5 (2005) 1677-1710

Published: 7 December 2005

ATG

\title{
Hopf diagrams and quantum invariants
}

\author{
Alain Bruguitìes \\ Alexis Virelizier
}

\begin{abstract}
The Reshetikhin-Turaev invariant, Turaev's TQFT, and many related constructions rely on the encoding of certain tangles ( $n$-string links, or ribbon $n$-handles) as $n$-forms on the coend of a ribbon category. We introduce the monoidal category of Hopf diagrams, and describe a universal encoding of ribbon string links as Hopf diagrams. This universal encoding is an injective monoidal functor and admits a straightforward monoidal retraction. Any Hopf diagram with $n$ legs yields a $n$-form on the coend of a ribbon category in a completely explicit way. Thus computing a quantum invariant of a 3-manifold reduces to the purely formal computation of the associated Hopf diagram, followed by the evaluation of this diagram in a given category (using in particular the so-called Kirby elements).
\end{abstract}

AMS Classification 57M27; 18D10, 81R50

Keywords Hopf diagrams, string links, quantum invariants

\section{Introduction}

In 1991, Reshetikhin and Turaev 12 introduced a new 3-manifold invariant. The construction proceeds in two steps: representing a 3-manifold by surgery along a link and then coloring the link to obtain a scalar invariant. Here, colors are (linear combinations of) simple representations of a quantum group at a root of unity. Since then, this construction has been re-visited many times.

In particular, Turaev 13] introduced the notion of a modular category, which is (after innocuous additivisation and karoubianisation, see [1]) an abelian semisimple ribbon category satisfying a finiteness and a non-degeneracy condition, and showed that such a category defines a 3-manifold invariant, and indeed a TQFT. In this approach, colors are simple objects of the category.

Following these ideas, a more general approach on quantum invariants of 3-manifolds has been subsequently developed, see [7] and more recently [3, 14]. It avoids in particular the semisimplicity condition. Let us briefly outline it: the 
initial data used to construct the invariants is a ribbon category $\mathcal{C}$ endowed with a coend $A=\int^{X \in \mathcal{C}} \vee_{X} \otimes X$. Let $L$ be a framed $n$-link. We can always present $L$ as the closure of some ribbon $n$-string link $T$. By using the universal property of the coend $A$, to such a string link $T$ is associated a $n$-form on $A$, that is, a morphism $T_{\mathcal{C}}: A^{\otimes n} \rightarrow \mathbf{1}$ in $\mathcal{C}$. Given a morphism $\alpha: \mathbf{1} \rightarrow A$ (which plays here the role of the color), set:

$$
\tau_{\mathcal{C}}(L ; \alpha)=T_{\mathcal{C}} \circ \alpha^{\otimes n} \in \operatorname{End}_{\mathcal{C}}(\mathbf{1}) .
$$

A Kirby element of $\mathcal{C}$, as defined in [14, is a morphism $\alpha: \mathbf{1} \rightarrow A$ such that, for all framed link $L, \tau_{\mathcal{C}}(L ; \alpha)$ is well-defined and invariant under isotopies of $L$ and under 2-handle slides. In this case, by Kirby's theorem [4] and under some invertibility condition, the invariant $\tau_{\mathcal{C}}(L ; \alpha)$ can be normalized to an invariant of 3-manifolds.

At this stage, two main questions naturally arise. Firstly, how to recognize the Kirby elements of a ribbon category? And secondly, how to compute the forms $T_{\mathcal{C}}$ obtained via the universal property?

Concerning the first question, recall that the coend $A$ has a structure of a Hopf algebra in the category $\mathcal{C}$, see 9 , 6]. This means in particular that $A$ is endowed with a product $\mu_{A}: A \otimes A \rightarrow A$, a coproduct $\Delta_{A}: A \rightarrow A \otimes A$, and an antipode $S_{A}: A \rightarrow A$ which satisfy the same axioms as those of a Hopf algebra except one has to replace the usual flip map with the braiding of $\mathcal{C}$. If $A$ admits a two-sided integral $\lambda: \mathbf{1} \rightarrow A$, then $\lambda$ is a Kirby element and the corresponding 3-manifold invariant is that of Lyubashenko [7]. More generally, if a morphism $\alpha: \mathbf{1} \rightarrow A$ in $\mathcal{C}$ satisfies:

$$
\left(\operatorname{id}_{A} \otimes \mu_{A}\right)\left(\Delta_{A} \otimes \operatorname{id}_{A}\right)(\alpha \otimes \alpha)=\alpha \otimes \alpha \quad \text { and } \quad S_{A} \alpha=\alpha,
$$

then it is a Kirby element, see [14. In particular, any Reshetikhin-Turaev invariant computed from a semisimple sub-quotient of $\mathcal{C}$ can be defined directly via a Kirby element of $\mathcal{C}$ satisfying this last equation.

The main motivation of the present paper is to answer the second question. Given a ribbon string link $T$, we express $T_{\mathcal{C}}$ in terms of some structural morphisms of $A$ (avoiding the product). This can be done by means of a universal formula, that is, independently of $\mathcal{C}$. To this end, we introduce the notion of Hopf diagrams. They are braided planar diagrams (with inputs but no

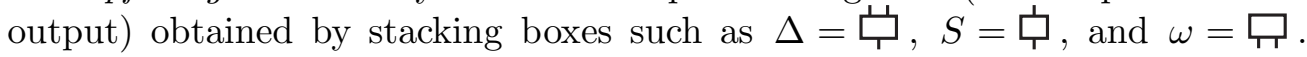
These are submitted to relations corresponding to those of a coproduct, an antipode, and a Hopf pairing. To each Hopf diagram $D$ with $n$ inputs is associated a ribbon $n$-string link $\psi(D)$ as in Figure 1. We construct a category $\overline{\mathcal{D}} \boldsymbol{i a g}^{S}$, whose objects are non-negative integers and morphisms are Hopf 


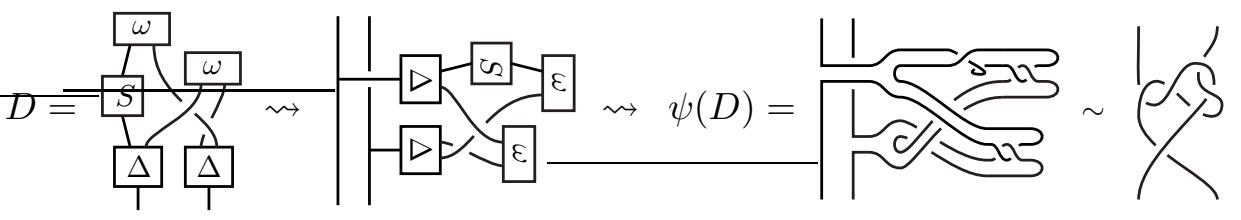

Figure 1: From Hopf diagrams to ribbon string links

diagrams up to a certain equivalence relation, in such a way that $\psi$ factors as a monoidal functor $\bar{\psi}$ from $\overline{\mathcal{D}} i a g^{S}$ to the category RSL of ribbon string links. Moreover we construct a monoidal functor $\Psi: \mathrm{RSL} \rightarrow \overline{\mathcal{D} i a g}$ S which is right inverse to $\bar{\psi}$, that is, such that $\bar{\psi} \circ \Psi=1_{\mathrm{RSL}}$. This is the main result of this paper (Theorem 4.5). This monoidal functor may be viewed as a formal encoding of ribbon string links. More precisely, given a ribbon category $\mathcal{C}$ endowed with a coend $A=\int^{X \in \mathcal{C}}{ }^{\vee} X \otimes X$, there is a canonical map $E_{\mathcal{C}}:\{$ Hopf diagrams with $n$ inputs $\} \rightarrow \operatorname{Hom}_{\mathcal{C}}\left(A^{\otimes n}, \mathbf{1}\right)$ such that $T_{\mathcal{C}}=$ $E_{\mathcal{C}} \circ \Psi(T)$ for any ribbon $n$-string link $T$ (see Theorem [5.1).

In Section 1, we define the the monoidal category $\operatorname{Diag}^{S}$ of Hopf diagrams, as a convolution category. The category of Hopf diagrams comes in two versions: with, or without antipode. Both versions are isomorphic, however. In Section 2 we review the monoidal category RSL of ribbon string links and the related monoidal category of ribbon handles. These categories are isomorphic. In Section 3. we construct a monoidal functor $\psi: \mathcal{D} i a g^{S} \rightarrow$ RSL. In Section 4, we define a category $\overline{\mathcal{D} i a g}$, as a quotient of $\mathcal{D} i a g^{S}$ by certain new relations, in such a way that $\psi$ induces a monoidal functor $\bar{\psi}: \overline{\mathcal{D} i a g}{ }^{S} \rightarrow$ RSL which admits

a right inverse. Finally, in Section 5 , given a ribbon category $\mathcal{C}$ endowed with a coend $A$, we explain how to represent the category of Hopf diagrams into $\mathcal{C}$ by using some structural morphisms of $A$. Moreover, we give a general criterion, using Hopf diagrams, for recognizing Kirby elements.

Unless otherwise specified, monoidal categories are assumed to be strict.

Acknowledgements This work has been partially funded by the CNRS-NSF project $\mathrm{n}^{\circ} 17149$ 'Algebraic and homologic methods in low dimensional topology'. The second author thanks the Max-Planck-Institut für Mathematik in Bonn, where part of this work was carried out, for its support and hospitality.

\section{Hopf diagrams}

In this section, we construct the categories Diag and Diag $^{S}$ (as convolution categories) which are shown to be isomorphic. They are preliminary versions 
of the category of Hopf diagrams.

\subsection{Categorical (co)algebras}

Recall that an algebra in a monoidal category is an object $A$ endowed with morphisms $\mu: A \otimes A \rightarrow A$ (the product) and $\eta: A \rightarrow \mathbf{1}$ (the unit) which satisfy:

$$
\mu\left(\operatorname{id}_{A} \otimes \mu\right)=\mu\left(\mu \otimes \operatorname{id}_{A}\right) \quad \text { and } \quad \mu\left(\eta \otimes \operatorname{id}_{A}\right)=\operatorname{id}_{A}=\mu\left(\operatorname{id}_{A} \otimes \eta\right) .
$$

Dually, a coalgebra in a monoidal category is an object $C$ endowed with morphisms $\Delta: C \rightarrow C \otimes C$ (the coproduct) and $\varepsilon: C \rightarrow \mathbf{1}$ (the counit) which satisfy:

$$
\left(\operatorname{id}_{C} \otimes \Delta\right) \Delta=\left(\Delta \otimes \operatorname{id}_{C}\right) \Delta \quad \text { and } \quad\left(\varepsilon \otimes \operatorname{id}_{C}\right) \Delta=\operatorname{id}_{C}=\left(\operatorname{id}_{C} \otimes \varepsilon\right) \Delta .
$$

Note that the unit object $\mathbf{1}$ of a monoidal category $\mathcal{C}$ is both an algebra (with $\left.\mu=\mathrm{id}_{\mathbf{1}}=\eta\right)$ and a coalgebra (with $\left.\Delta=\mathrm{id}_{\mathbf{1}}=\varepsilon\right)$ in $\mathcal{C}($ recall that $\mathbf{1}=\mathbf{1} \otimes \mathbf{1})$.

When the category $\mathcal{C}$ is braided with braiding $\tau$, the monoidal product $A \otimes A^{\prime}$ of two algebras $A$ and $A^{\prime}$ in $\mathcal{C}$ is an algebra in $\mathcal{C}$ with unit $\eta \otimes \eta^{\prime}$ and product $\left(\mu \otimes \mu^{\prime}\right)\left(\operatorname{id}_{A} \otimes \tau_{A, A^{\prime}} \otimes \mathrm{id}_{A^{\prime}}\right)$. In particular, for any non-negative integer $n, A^{\otimes n}$ is an algebra in $\mathcal{C}$. Likewise, the monoidal product $C \otimes C^{\prime}$ of two coalgebras $C$ and $C^{\prime}$ in a braided category $\mathcal{C}$ is a coalgebra in $\mathcal{C}$ with counit $\varepsilon \otimes \varepsilon^{\prime}$ and coproduct $\left(\operatorname{id}_{C} \otimes \tau_{C, C^{\prime}} \otimes \operatorname{id}_{C^{\prime}}\right)\left(\Delta \otimes \Delta^{\prime}\right)$. In particular, for any non-negative integer $n, C^{\otimes n}$ is a coalgebra in $\mathcal{C}$.

\subsection{The convolution product}

Let $\mathcal{C}$ be a monoidal category, $(A, \mu, \eta)$ be an algebra in $\mathcal{C}$, and $(C, \Delta, \varepsilon)$ be a coalgebra in $\mathcal{C}$. The convolution product of two morphisms $f, g \in \operatorname{Hom}_{\mathcal{C}}(C, A)$ is the morphism $f \star g=\mu(f \otimes g) \Delta \in \operatorname{Hom}_{\mathcal{C}}(C, A)$. This makes the set $\operatorname{Hom}_{\mathcal{C}}(C, A)$ a monoid with unit $\eta \varepsilon: C \rightarrow A$.

\subsection{Convolution categories}

Let $\mathcal{C}$ be a braided category, $A$ be an algebra in $\mathcal{C}$, and $C$ be a coalgebra in $\mathcal{C}$. Let us define the convolution category $\operatorname{Conv}_{\mathcal{C}}(C, A)$ as follows: the objects of $\operatorname{Conv}_{\mathcal{C}}(C, A)$ are the non-negative integers $\mathbb{N}$. For $m, n \in \mathbb{N}$, the set of morphisms from $m$ to $n$ is empty if $m \neq n$ and is the monoid $\operatorname{Hom}_{\mathcal{C}}\left(C^{\otimes n}, A\right)$ 
endowed with the convolution product if $m=n$ (recall indeed that $C^{\otimes n}$ is a coalgebra in $\mathcal{C}$ ). In particular, the identity of an object $n \in \mathbb{N}$ is:

$$
\operatorname{id}_{n}=\eta \varepsilon^{\otimes n}: C^{\otimes n} \rightarrow A,
$$

and the composition of two endomorphisms $f, g \in \operatorname{Hom}_{\mathcal{C}}\left(C^{\otimes n}, A\right)$ of an object $n \in \mathbb{N}$ is given by the convolution product:

$$
f \circ g=f \star g=\mu(f \otimes g) \Delta_{C} \otimes n: C^{\otimes n} \rightarrow A,
$$

where $\Delta_{C} \otimes n$ denotes the coproduct of the coalgebra $C^{\otimes n}$.

Note that the category $\operatorname{Conv}_{\mathcal{C}}(C, A)$ is a monoidal category: the monoidal product of two objects $m, n \in \mathbb{N}$ is given by $m \otimes n=m+n$, the unit object is $0 \in \mathbb{N}$, and the monoidal product of two morphisms $f: m \rightarrow m$ and $g: n \rightarrow n$ (where $m, n \in \mathbb{N}$ ) is the morphism $f \otimes g=\mu\left(f \otimes_{\mathcal{C}} g\right): m+n \rightarrow m+n$.

\subsection{The category Diag}

Let $\mathcal{D}_{0}$ be the braided category freely generated by one object $*$ and the following morphisms:

$$
\begin{array}{lll}
\Delta: * \rightarrow * \otimes *, & \omega_{+}: * \otimes * \rightarrow 1, & \theta_{+}: * \rightarrow \mathbf{1}, \\
\varepsilon: * \rightarrow 1, & \omega_{-}: * \otimes * \rightarrow 1, & \theta_{-}: * \rightarrow \mathbf{1},
\end{array}
$$

where 1 denotes the unit object of the monoidal product. Let $\mathcal{D}$ be the quotient of the category $\mathcal{D}_{0}$ by the following relations:

$$
\begin{aligned}
& \left(\mathrm{id}_{*} \otimes \Delta\right) \Delta=\left(\Delta \otimes \mathrm{id}_{*}\right) \Delta, \\
& \left(\mathrm{id}_{*} \otimes \varepsilon\right) \Delta=\mathrm{id}_{*}=\left(\varepsilon \otimes \mathrm{id}_{*}\right) \Delta .
\end{aligned}
$$

The category $\mathcal{D}$ is still braided (with induced braiding) and $(*, \Delta, \varepsilon)$ is a coalgebra in $\mathcal{D}$. We define the category $\mathcal{D}$ iag to be the convolution category $\operatorname{Conv}_{\mathcal{D}}(*, \mathbf{1})$, see Section 1.3. where $\mathbf{1}$ is endowed with the trivial algebra structure.

\subsection{The category Diag $^{S}$}

Let $\mathcal{D}_{0}^{S}$ be the braided category freely generated by one object $*$ and the following morphisms:

$$
\begin{array}{lll}
\Delta: * \rightarrow * \otimes *, & \omega_{+}: * \otimes * \rightarrow \mathbf{1}, & \theta_{+}: * \rightarrow \mathbf{1}, \\
\varepsilon: * \rightarrow \mathbf{1}, & \omega_{-}: * \otimes * \rightarrow \mathbf{1}, & \theta_{-}: * \rightarrow \mathbf{1}, \\
S: * \rightarrow *, & S^{-1}: * \rightarrow * . &
\end{array}
$$


Let $\mathcal{D}^{S}$ be the quotient of the category $\mathcal{D}_{0}^{S}$ by the relations (11) and (2), and the following relations:

$$
\begin{aligned}
& S S^{-1}=\mathrm{id}_{*}=S^{-1} S, \\
& \Delta S=(S \otimes S) \tau_{*, *} \Delta, \\
& \varepsilon S=\varepsilon \\
& \theta_{ \pm} S=\theta_{ \pm}, \\
& \omega_{+}\left(S \otimes \mathrm{id}_{*}\right)=\omega_{-}=\omega_{+}\left(\mathrm{id}_{*} \otimes S\right), \\
& \omega_{+}\left(S^{-1} \otimes \mathrm{id}_{*}\right)=\omega_{-} \tau_{*, *}=\omega_{+}\left(\mathrm{id}_{*} \otimes S^{-1}\right),
\end{aligned}
$$

where $\tau_{*, *}: * \otimes * \rightarrow * \otimes *$ denotes the braiding of the object $*$ with itself in $\mathcal{D}_{0}^{S}$.

The category $\mathcal{D}^{S}$ is still braided (with induced braiding) and $(*, \Delta, \varepsilon)$ is a coalgebra in $\mathcal{D}^{S}$. We define the category $\mathcal{D} \mathrm{ig}^{S}$ to be the convolution category $\operatorname{Conv}_{\mathcal{D}^{S}}(*, \mathbf{1})$, see Section 1.3, where $\mathbf{1}$ is endowed with the trivial algebra structure.

\subsection{Relations between $\mathcal{D} i a g$ and $\mathcal{D}_{i a g}{ }^{S}$}

The inclusion functor $\mathcal{D}_{0} \hookrightarrow \mathcal{D}_{0}^{S}$ induces a functor $\mathcal{D} \rightarrow \mathcal{D}^{S}$ and so a functor $\iota: \mathcal{D}$ iag $\rightarrow \mathcal{D}$ iag ${ }^{S}$. Note that $\iota$ is the identity on the objects.

Theorem 1.1 $\iota: \mathcal{D} i a g \rightarrow \mathcal{D} i a g^{S}$ is an isomorphism of categories.

Proof Fix $n \in \mathbb{N}$. Set $C=\operatorname{Hom}_{\mathcal{D}_{0}}\left(*^{n}, *\right)$ and $C^{S}=\operatorname{Hom}_{\mathcal{D}_{0}^{S}}\left(*^{n}, *\right)$. We identify $C$ with its image under the functor $\mathcal{D}_{0} \hookrightarrow \mathcal{D}_{0}^{S}$, so that we have $C \subset C^{S}$. Let $\sim$ be the equivalence relation on $C$ defined by (11)-(2), and let $\sim^{S}$ be the equivalence relation on $C^{S}$ defined by (11)-(8). All we have to show is that the map $F: C / \sim \rightarrow C^{S} / \sim^{S}$ induced by the inclusion $C \hookrightarrow C^{S}$ is bijective.

We will construct a map $C^{S} \rightarrow C, f \mapsto f^{\prime}$, satisfying:

(a) $f^{\prime} \sim^{S} f$ for all $f \in C^{S}$;

(b) $f^{\prime}=f$ for all $f \in C$;

(c) $f \sim^{S} g \Longrightarrow f^{\prime} \sim g^{\prime}$ for all $f, g \in C^{S}$.

If this holds, then $F$ is onto by (a), and $F$ is into by (b) and (c). 
Now consider the following rewriting rules:

$$
\begin{array}{ll}
\Delta S^{ \pm 1} \longrightarrow\left(S^{ \pm 1} \otimes S^{ \pm 1}\right) \tau_{*, *}^{ \pm 1} \Delta, & \epsilon S^{ \pm 1} \longrightarrow \epsilon, \\
S S^{-1} \longrightarrow \mathrm{id}_{*}, & S^{-1} S \longrightarrow \mathrm{id}_{*}, \\
\theta_{+} S^{ \pm 1} \longrightarrow \theta_{+}, & \theta_{-} S^{ \pm 1} \longrightarrow \theta_{-}, \\
\omega_{+}\left(S \otimes \mathrm{id}_{*}\right) \longrightarrow \omega_{-}, & \omega_{+}\left(\mathrm{id}_{*} \otimes S\right) \longrightarrow \omega_{-}, \\
\omega_{+}\left(S^{-1} \otimes \mathrm{id}_{*}\right) \longrightarrow \omega_{-} \tau_{*, *}, & \omega_{+}\left(\mathrm{id}_{*} \otimes S^{-1}\right) \longrightarrow \omega_{-} \tau_{*, *}, \\
\omega_{-}\left(S \otimes \mathrm{id}_{*}\right) \longrightarrow \omega_{+} \tau_{*, *}^{-1}, & \omega_{-}\left(\mathrm{id}_{*} \otimes S\right) \longrightarrow \omega_{+} \tau_{*, *}^{-1}, \\
\omega_{-}\left(S^{-1} \otimes \mathrm{id}_{*}\right) \longrightarrow \omega_{+}, & \omega_{-}\left(\mathrm{id}_{*} \otimes S^{-1}\right) \longrightarrow \omega_{+} .
\end{array}
$$

For $f, g \in C^{S}$, we write $f \leq g$ (resp. $f \prec g$ ) if we can go from $f$ to $g$ by applying a finite number (resp. exactly one) of these rules. The rewriting system is noetherian. Indeed, the first rule decreases the number of letters $S^{ \pm 1}$ on the right of $\Delta$, and all other rules decrease the number of letters $S^{ \pm 1}$. Therefore $\left(C^{S}, \leq\right)$ is a noetherian partially ordered set. Moreover the system is confluent: if $x \prec y$ and $x \prec z$, then there exists $t$ such that $y, z \leq t$. This can easily be checked by considering all cases when two rewriting rules can be applied to the same element $f \in C^{S}$. By [11, for each $f \in C^{S}$ there exists a unique maximal element $f^{\prime} \in C^{S}$ such that $f \leq f^{\prime}$. The maximality condition means that no rewriting rule can be applied to $f^{\prime}$. Such can only be the case if no letter $S^{ \pm 1}$ occurs in $f^{\prime}$, that is, if $f^{\prime} \in C$.

So we have constructed a map $f \mapsto f^{\prime}$. Condition (b) is obvious. Condition (a) results from the observation that $\sim^{S}$ is the equivalence relation on $C^{S}$ generated by $\prec$ and (11)-(2). Let us check Condition (c). By construction, if $f \prec g$ then $f^{\prime}=g^{\prime}$. So, by the previous observation, it is enough to verify that if $g$ is obtained from $f$ by a modification of the form (1) or (2), then $f^{\prime} \sim g^{\prime}$. This is easily shown by noetherian induction. The only point here is to verify that, in the presence of a configuration of the type $\left(\Delta \otimes \mathrm{id}_{*}\right) \Delta S^{ \pm 1}$, applying (1) followed by twice the first rewriting rule gives the same result as applying twice the first rewriting rule followed by (11). Hence the theorem.

\subsection{Hopf diagrams}

By a Hopf diagram, we shall mean a morphism of Diag or Diag ${ }^{S}$. Hopf diagrams can be represented by plane diagrams: we draw one of their preimage in the braided category $\mathcal{D}$ or $\mathcal{D}^{S}$ by using Penrose graphical calculus with the ascending convention (diagrams are read from bottom to top) as for instance 
in [13. We depict the generators as in Figure 2 except Figure 2(e) which depicts $\Delta^{(n)}: * \rightarrow *^{\otimes(n+1)}$ defined inductively by:

$$
\Delta^{(0)}=\mathrm{id}_{*}, \quad \Delta^{(1)}=\Delta \quad \text { and } \quad \Delta^{(n+1)}=\left(\Delta^{(n)} \otimes \mathrm{id}_{*}\right) \Delta .
$$

The relations defining $\mathcal{D}_{i a g}{ }^{S}$ (except those concerning the braiding) are depicted in Figure 3. Recall that the composition $D_{1} \circ D_{2}=D_{1} \star D_{2}$ of two Hopf diagrams $D_{1}$ and $D_{2}$ is given by the convolution product, see Figure 4

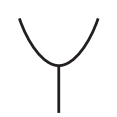

(a) $\Delta: * \rightarrow * \otimes *$

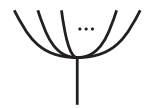

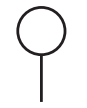

(b) $\varepsilon: * \rightarrow \mathbf{1}$

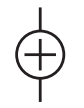

(c) $S: * \rightarrow *$

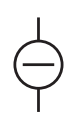

(d) $S^{-1}: * \rightarrow *$

(e) $\Delta^{(n)}: * \rightarrow *^{\otimes(n+1)}$
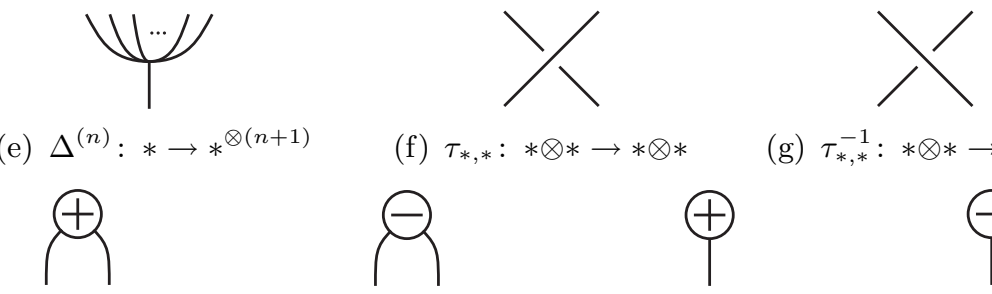

(f) $\tau_{*, *}: * \otimes * \rightarrow * \otimes *$

(g) $\tau_{*, *}^{-1}: * \otimes * \rightarrow * \otimes *$
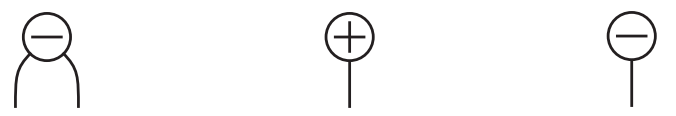

(h) $\omega_{+}: * \otimes * \rightarrow \mathbf{1}$

(i) $\omega_{-}: * \otimes * \rightarrow \mathbf{1}$

(j) $\theta_{+}: * \rightarrow \mathbf{1}$

(k) $\theta_{-}: * \rightarrow \mathbf{1}$

Figure 2: Generators of $\operatorname{Diag}^{S}$
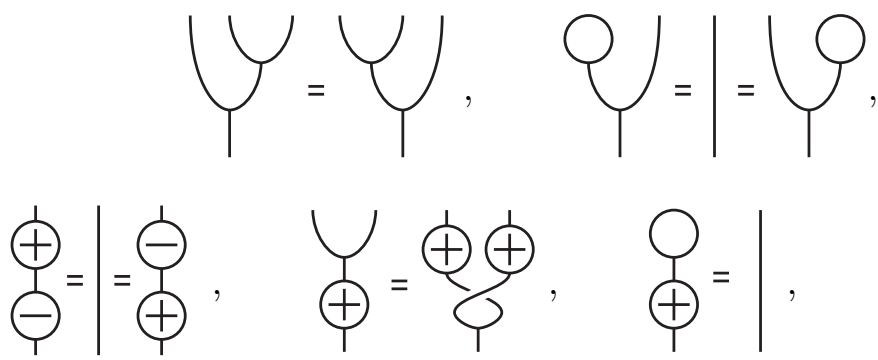

$\Psi=\theta_{0}^{+}$

$Y_{1}=$

$\stackrel{\oplus}{\oplus}=I^{ \pm}$

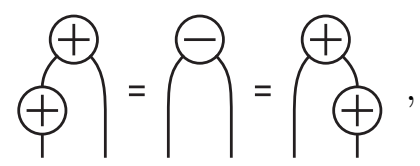

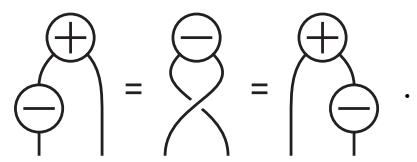

Figure 3: Relations in $\operatorname{Diag}^{S}$

\section{Ribbon string links and ribbon handles}

As usual, we represent ribbon tangles (also called framed tangles) by thin plane diagrams (using blackboard framing). Recall that two such diagrams represent 


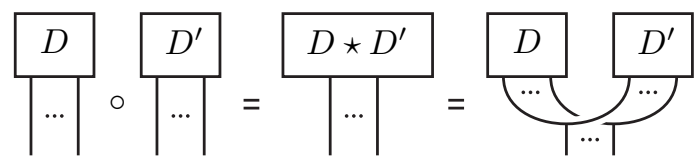

Figure 4: Composition in $\mathcal{D i a g}^{S}$

the same isotopy class of a ribbon tangle if and only if one can be obtained from the other by deformation and a finite sequence of ribbon Reidemeister moves depicted in Figure 5

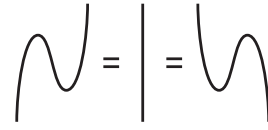

(a) Type 0

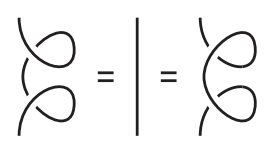

(b) Type $1^{\prime}$

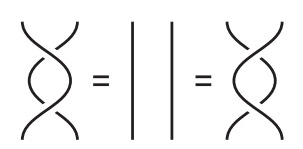

(c) Type 2

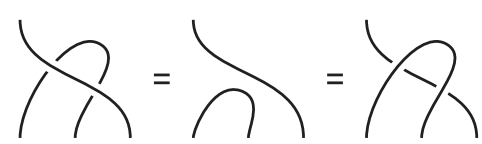

(d) Type $2^{\prime}$

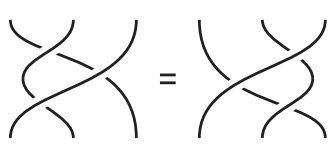

(e) Type 3

Figure 5: Ribbon Reidemeister moves

\subsection{Ribbon string links}

Let $n$ be a non-negative integer. By a ribbon $n$-string link we shall mean a ribbon $(n, n)$-tangle $T \subset \mathbb{R}^{2} \times[0,1]$ consisting of $n$ arc components, without any closed component, such that the $k$ th arc $(1 \leq k \leq n)$ joins the $k$ th bottom endpoint to the $k$ th top endpoint. Note that a ribbon string link is canonically oriented by orienting each component from bottom to top.

We denote by RSL the category of ribbon string links. The objects of RSL are the non-negative integers. For two non-negative integers $m$ and $n$, the set of morphisms from $m$ to $n$ is

$$
\operatorname{Hom}_{\mathrm{RSL}}(m, n)= \begin{cases}\emptyset & \text { if } m \neq n, \\ \operatorname{RSL}_{n} & \text { if } m=n,\end{cases}
$$

where $\mathrm{RSL}_{n}$ denotes the set of (isotopy classes) of ribbon $n$-string links. The composition $T^{\prime} \circ T$ of two ribbon $n$-string links is given by stacking $T^{\prime}$ on the top of $T$ (i.e., with ascending convention). Identities are the trivial string links. 
Note that the category RSL is a monoidal category: $m \otimes n=m+n$ on objects and the monoidal product $T \otimes T^{\prime}$ of two ribbon string links $T$ and $T^{\prime}$ is the ribbon string link obtained by juxtaposing $T$ on the left of $T^{\prime}$ (see, e.g., [13).

\subsection{Ribbon handles}

Let $n$ be a non-negative integer. By a ribbon $n$-handle we shall mean a ribbon $(2 n, 0)$-tangle $T \subset \mathbb{R}^{2} \times[0,1]$ consisting of $n$ arc components, without any closed component, such that the $k$-th arc $(1 \leq k \leq n)$ joins the $(2 k-1)$-th bottom endpoint to the $2 k$-th bottom endpoint. Note that a ribbon handle is canonically oriented by orienting each component upwards near its right bottom input.

We denote by RHand the category of ribbon handles. The objects of RHand are the non-negative integers. For two non-negative integers $m$ and $n$, the set of morphisms from $m$ to $n$ is

$$
\operatorname{Hom}_{\text {RHand }}(m, n)= \begin{cases}\emptyset & \text { if } m \neq n, \\ \operatorname{RHand}_{n} & \text { if } m=n,\end{cases}
$$

where $\mathrm{RHand}_{n}$ denotes the set of (isotopy classes) of ribbon $n$-handles. The composition of two ribbon $n$-handles $T$ and $T^{\prime}$ is the ribbon $n$-handle defined in Figure 6(c) The identity for this composition consists in $n$ caps, see Figure 6(d).

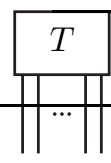

(a) $T$

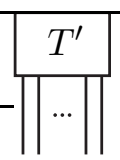

(b) $T^{\prime}$

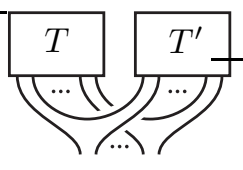

(c) $T \circ T^{\prime}$

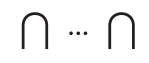

(d) id

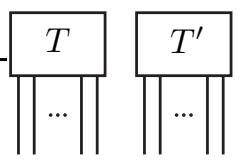

(e) $T \otimes T^{\prime}$

Figure 6: Composition, identity, and monoidal product in RHand

Note that the category RHand is a monoidal category: $m \otimes n=m+n$ on objects and the monoidal product $T \otimes T^{\prime}$ of two ribbon handles $T$ and $T^{\prime}$ is the ribbon handle obtained by juxtaposing $T$ on the left of $T^{\prime}$, see Figure 6(e)

\subsection{An isomorphism between ribbon handles and string links}

Let us construct functors $F:$ RSL $\rightarrow$ RHand and $G:$ RHand $\rightarrow$ RSL as follows. On objects, set $F(n)=n$ and $G(n)=n$ for any non-negative integer $n$. For any 
ribbon $n$-string link $S$, let $F(S)$ be the ribbon $n$-handle defined in Figure 7(b) For any ribbon $n$-handle $T$, let $G(T)$ be the ribbon $n$-string link defined in Figure $7(\mathrm{~d})$.

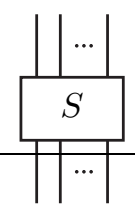

(a) $S$

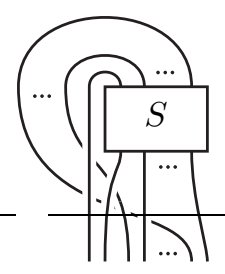

(b) $F(S)$

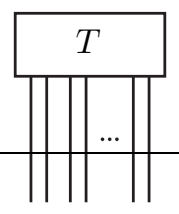

(c) $T$

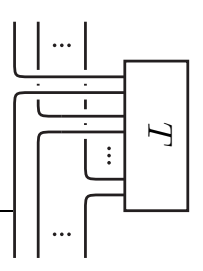

(d) $G(T)$

Figure 7: Definition of the functors $F$ and $G$

Proposition 2.1 The functors $F:$ RSL $\rightarrow$ RHand and $G$ : RHand $\rightarrow$ RSL are mutually inverse monoidal functors.

Proof Straightforward.

\section{From Hopf diagrams to ribbon string links}

\subsection{From Hopf diagrams to ribbon handles}

Let us define a functor $\phi$ from the category $\mathcal{D} i a g^{S}$ of Hopf diagrams to the category RHand of ribbon handles. For any non-negative integer $n$, we set $\phi(n)=n$. Given a Hopf diagram $D$, we construct a diagram of ribbon handle $\phi_{D}$ by using the rules of Figure 8 and the stacking product (with ascending convention). See Figure 9 for an example. Then let $\phi(D)$ be the isotopy class of the ribbon handles defined by $\phi_{D}$.

Proposition 3.1 The functor $\phi:$ Diag $^{S} \rightarrow$ RHand is well-defined and monoidal.

We will prove in Section 4 (see Corollary 4.6) that $\phi$ is surjective.

Proof Let us first verify that $\phi$ is well-defined. We only have to verify that both sides of the relations defining Diag $^{S}$ are transformed by the rules of Figure 8 to isotopic tangles. Examples of such verifications are depicted in Figure 10. The fact that $\phi$ is a monoidal functor comes from the definitions of composition and monoidal product in $\mathcal{D} i a g^{S}$ and RHand. 


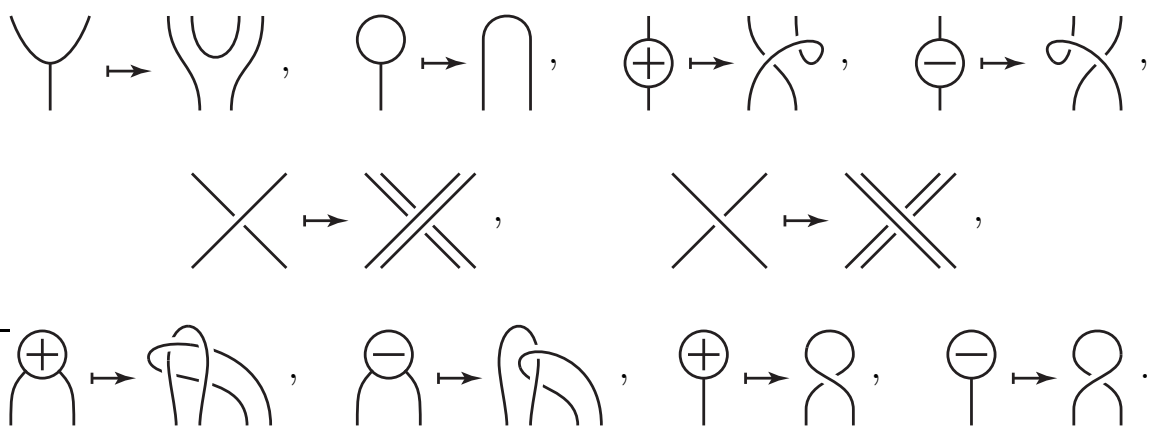

Figure 8: Rules for defining $\phi$

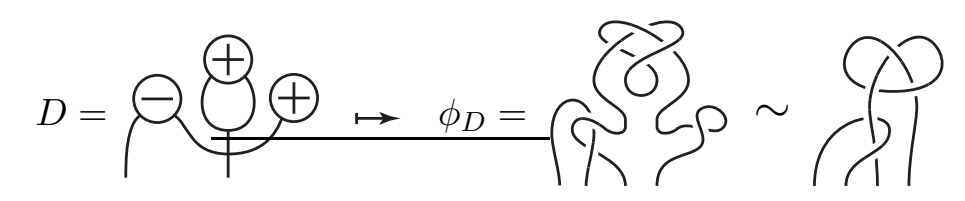

Figure 9
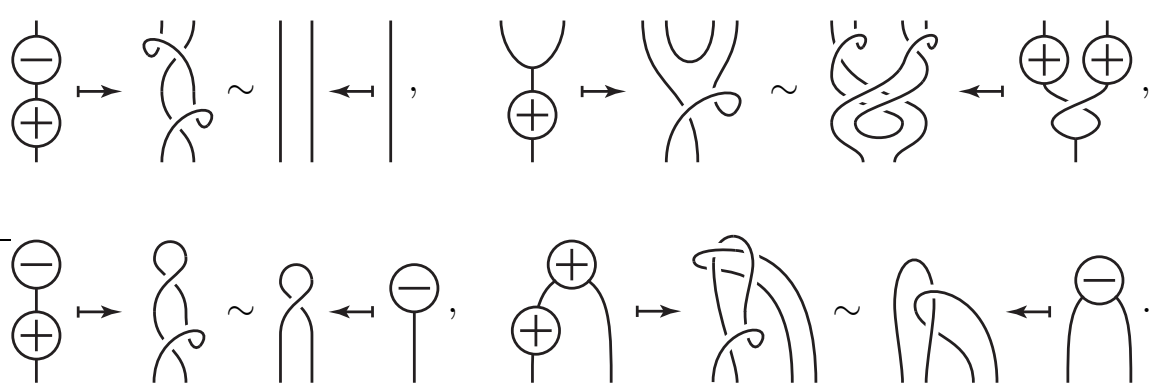

Figure 10

\subsection{From Hopf diagrams to ribbon string links}

Let us define a functor $\psi:$ Diag $^{S} \rightarrow$ RSL as follows. For any non-negative integer $n$, let $\psi(n)=n$. If $D$ is a Hopf diagram, we construct a diagram of ribbon string link $\psi_{D}$ as in Figure 11(a), where $\phi_{D}$ is defined as above. For an example, see Figure 11(b), Then we let $\psi(D)$ be the isotopy class of the ribbon string link defined by $\psi_{D}$.

Corollary 3.2 The functor $\psi:$ Diag ${ }^{S} \rightarrow$ RSL is well-defined and surjective. Moreover $F \circ \psi=\phi$ and $G \circ \phi=\psi$, where $F$ and $G$ are the functors of Section 2.3 .

Proof This is an immediate consequence of Propositions 2.1 and 3.1 


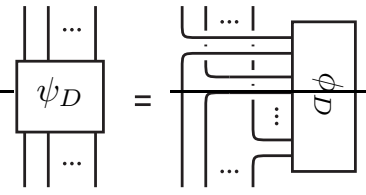

(a)

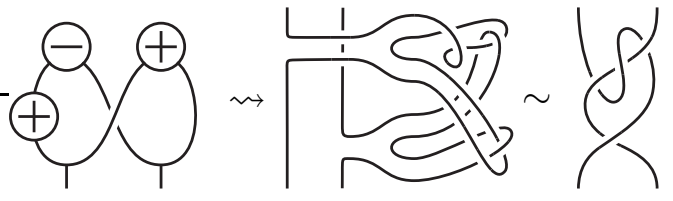

(b)

Figure 11

\section{From ribbon string links to Hopf diagrams}

In this section, we construct a refined version $\overline{\mathcal{D} i a g}$ S of the category of Hopf diagrams and we prove the main theorem.

\subsection{More relations on Hopf diagrams}

Recall the braided category $\mathcal{D}^{S}$ of Section 1.5. Let $\overline{\mathcal{D}}^{S}$ be the quotient of $\mathcal{D}^{S}$ by the following relations:

$$
\begin{aligned}
& \left(\mathrm{id}_{*} \otimes \theta_{ \pm}\right) \Delta=\left(\theta_{ \pm} \otimes \mathrm{id}_{*}\right) \Delta, \\
& \left(\theta_{+} \otimes \theta_{-}\right) \Delta=\varepsilon, \\
& \left(\theta_{+} \otimes \theta_{+}\right) \Delta=\omega_{+} \tau_{*, *}^{-1} \Delta, \\
& \left(\theta_{-} \otimes \theta_{-}\right) \Delta=\omega_{-} \Delta, \\
& \omega_{+} \star \omega_{-}=\varepsilon \otimes \varepsilon=\omega_{-} \star \omega_{+}, \\
& \omega_{+12} \star \omega_{+13} \star \omega_{+23}=\omega_{+13} \star \omega_{+23} \star \omega_{+12}=\omega_{+23} \star \omega_{+12} \star \omega_{+13}, \\
& \omega_{+13} \star \omega_{+23} \star \omega_{+24} \star \omega_{-23}=\omega_{+23} \star \omega_{+24} \star \omega_{-23} \star \omega_{+13},
\end{aligned}
$$

where $\star$ denotes the convolution product of Hopf diagrams. Graphically, these relations can be depicted as in Figure 12, The category $\overline{\mathcal{D}}^{S}$ is still braided (with induced braiding) and $(*, \Delta, \varepsilon)$ is a coalgebra in $\overline{\mathcal{D}}^{S}$. Then let $\overline{\mathcal{D} i a g}{ }^{S}$ be the convolution category $\operatorname{Conv}_{\overline{\mathcal{D}}^{S}}(*, \mathbf{1})$, see Section 1.3. where 1 is endowed with the trivial algebra structure. By abuse, we will still call Hopf diagrams the morphisms of $\overline{\mathcal{D} i a g}$.

Note that the category $\overline{\mathcal{D} i a g}{ }^{S}$ can also be viewed as the quotient of the category $\operatorname{Diag}^{S}$ by Relations (9)-(15). Let $\pi: \mathcal{D} i a g^{S} \rightarrow \overline{\mathcal{D}}$ iag $^{S}$ be the projection functor.

Proposition 4.1 The functors $\phi$ and $\psi$ factorize through $\pi$ to functors $\bar{\phi}$ and $\bar{\psi}$, respectively, so that $\bar{\phi}=F \circ \bar{\psi}$. 


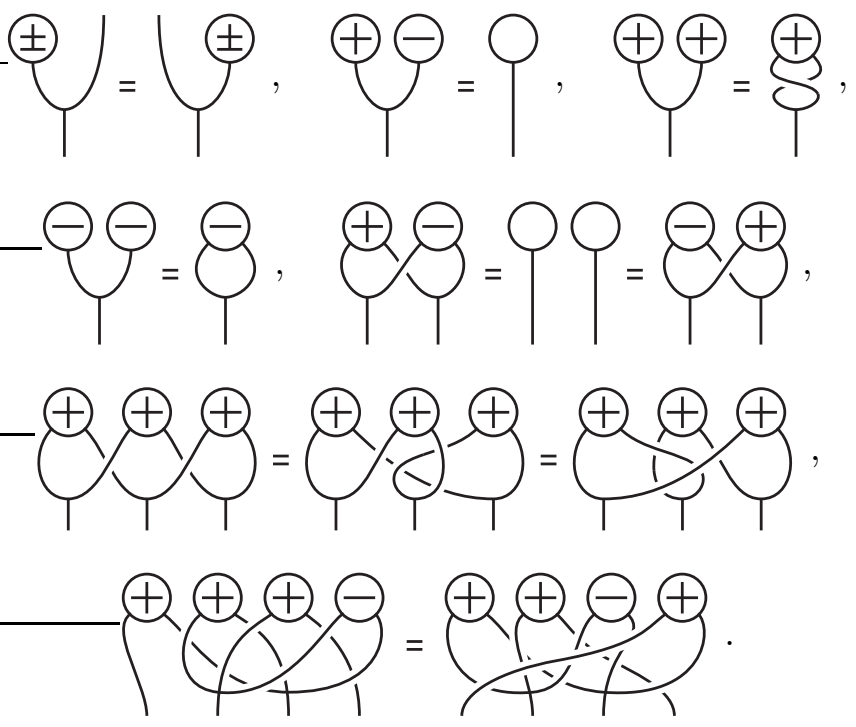

Figure 12

Proof We have to verify both sides of Relations (92)-(15) are transformed by the rules of Figure 8 to isotopic tangles. Examples of such verifications are depicted in Figure 13. Note that in the first picture, we used the fact that the $(2 i-1)$-th and $2 i$-th inputs of a ribbon handle are connected.

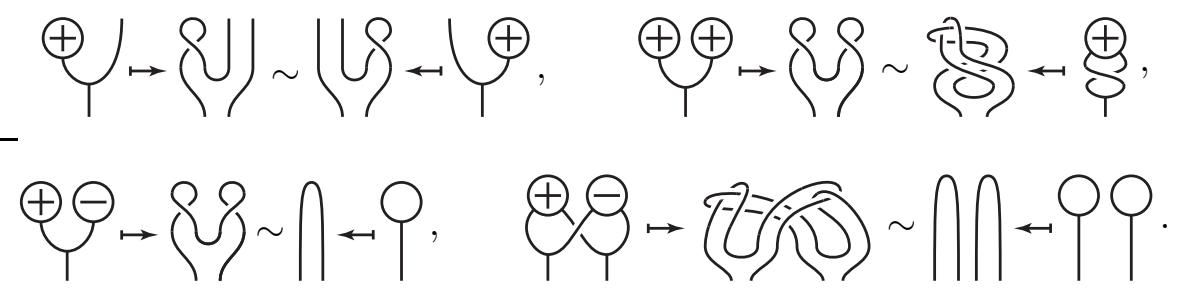

Figure 13

\subsection{From ribbon pure braids to Hopf diagrams}

Let RPB the subcategory of RSL made of ribbon pure braids. The objects of $\mathrm{RPB}$ are the non-negative integers. For two non-negative integers $m$ and $n$, 
the set of morphisms from $m$ to $n$ is

$$
\operatorname{Hom}_{\mathrm{RPB}}(m, n)= \begin{cases}\emptyset & \text { if } m \neq n, \\ \operatorname{RPB}_{n} & \text { if } m=n,\end{cases}
$$

where $\mathrm{RPB}_{n} \subset \mathrm{RSL}_{n}$ denotes the set of (isotopy classes) of ribbon pure $n$ braids. Note that RPB is a monoidal subcategory of RSL.

Recall we have a canonical group isomorphism:

$$
\left(u, t_{1}, \ldots, t_{n}\right): \mathrm{RPB}_{n} \stackrel{\sim}{\longrightarrow} \mathrm{PB}_{n} \times \mathbb{Z}^{n},
$$

where $\mathrm{PB}_{n}$ denotes the group of pure $n$-braids, $u: \mathrm{RPB}_{n} \rightarrow \mathrm{PB}_{n}$ is the forgetful morphism, and $t_{i}$ the self-linking number of the $i$-th component. Hence, using a presentation of $\mathrm{PB}_{n}$ by generators and relations due to Markov [10], we get that $\mathrm{RPB}_{n}$ is generated by $t_{k}(1 \leq k \leq n)$ and $\sigma_{i, j}(1 \leq i<j \leq n)$ subject to the following relations:

$$
\begin{aligned}
& t_{k} t_{l}=t_{l} t_{k} \quad \text { for any } k, l \\
& t_{k} \sigma_{i, j}=\sigma_{i, j} t_{k} \quad \text { for any } i<j \text { and } k ; \\
& \sigma_{i, j} \sigma_{k, l}=\sigma_{k, l} \sigma_{i, j} \quad \text { for any } i<j<k<l \text { or any } i<k<j<l ; \\
& \sigma_{i, j} \sigma_{i, k} \sigma_{j, k}=\sigma_{i, k} \sigma_{j, k} \sigma_{i, j}=\sigma_{j, k} \sigma_{i, j} \sigma_{i, k} \quad \text { for any } i<j<k ; \\
& \sigma_{i, k} \sigma_{j, k} \sigma_{j, l} \sigma_{j, k}^{-1}=\sigma_{j, k} \sigma_{j, l} \sigma_{j, k}^{-1} \sigma_{i, k} \quad \text { for any } i<j<k<l .
\end{aligned}
$$

Graphically, the generators may be represented as:

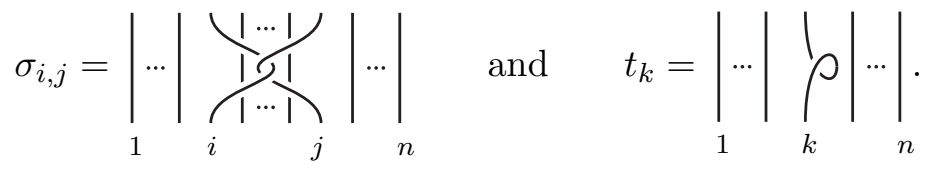

Let us define a functor $\Psi_{0}: \mathrm{RPB} \rightarrow \overline{\mathcal{D}}$ iag $^{S}$ as follows: for any non-negative integer $n$ set $\Psi(n)=n$. For $1 \leq i<j \leq n$ and $1 \leq k \leq n$, set

$$
\Psi_{0}\left(\sigma_{i, j}^{ \pm 1}\right)=\Sigma_{i, j}^{ \pm 1} \quad \text { and } \quad \Psi_{0}\left(t_{k}^{ \pm 1}\right)=\Omega_{k}^{ \pm 1},
$$

where

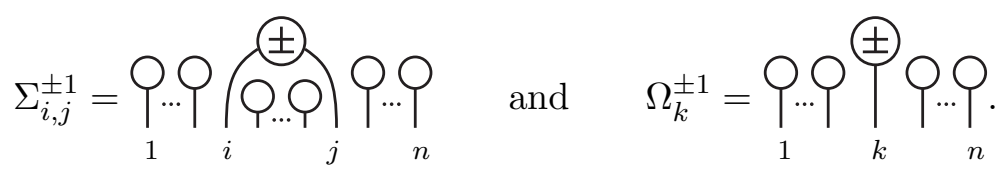

Lemma 4.2 The functor $\Psi_{0}: \mathrm{RPB} \rightarrow \overline{\mathcal{D}} \boldsymbol{i a g}^{S}$ is well defined, monoidal, and is such that $\bar{\psi} \circ \Psi_{0}(P)=P$ for all ribbon pure braid $P$. 
Proof Firstly, from Relation (13) (resp. Relations (9) and (10)), we see that the Hopf diagrams $\Sigma_{i, j}^{-1}$ and $\Sigma_{i, j}$ (resp. $\Omega_{k}^{-1}$ and $\Omega_{k}$ ) are inverse each other.

Secondly Relations (16)-(20) hold in $\overline{\mathcal{D}} \boldsymbol{1 a g}^{S}$, where we replace $\sigma_{i, j}$ and $t_{k}$ with $\Sigma_{i, j}$ and $\Omega_{k}$ respectively. Indeed Relations (16) and (17) follow from (9). Relation (18) follows from (2). Relations (19) and (20) correspond to (14) and (15) respectively.

Finally the isotopies depicted in Figure 14 show that $\bar{\psi} \circ \Psi_{0}\left(\sigma_{i, j}\right)=\sigma_{i, j}$ and $\bar{\psi} \circ \Psi_{0}\left(t_{k}\right)=t_{k}$. Hence $\bar{\psi} \circ \Psi_{0}(P)=P$ for all ribbon pure braid $P$.

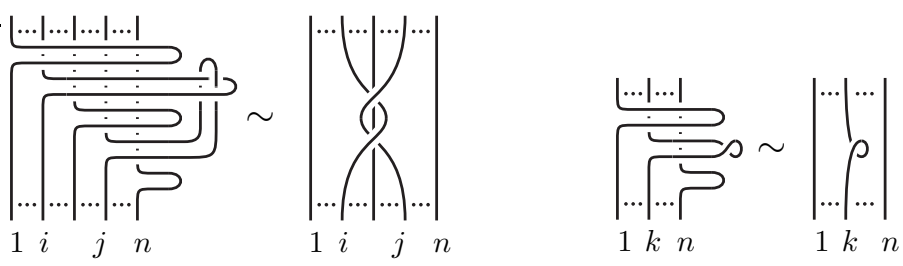

Figure 14

\subsection{Contractions}

Let $n \geq 3$ and $1<i<n$. For a ribbon $n$-string link $T$, we define the $i$-th contraction of $T$ to be the ribbon $(n-2)$-string link $c_{i}(T)$ defined as in Figure 15(a) For a Hopf diagram $D$ with $n$ inputs, we define the $i$-th contraction of $D$ to be the Hopf diagram with $(n-2)$ inputs $C_{i}(D)$ defined as in Figure 15(b)

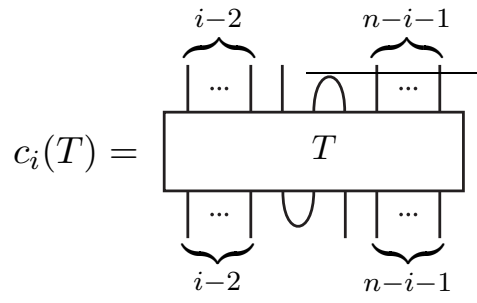

(a)

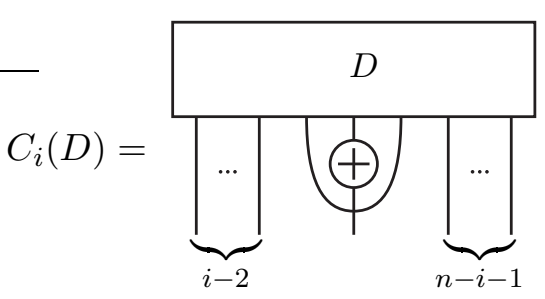

(b)

Figure 15: Contractions of ribbon string links and Hopf diagrams

Lemma 4.3 Let $n \geq 3$ and $1<i<n$. For any Hopf diagram $D$ with $n$ inputs, we have $c_{i}(\psi(D))=\psi\left(C_{i}(D)\right)$. 
Proof This follows from the following equalities:

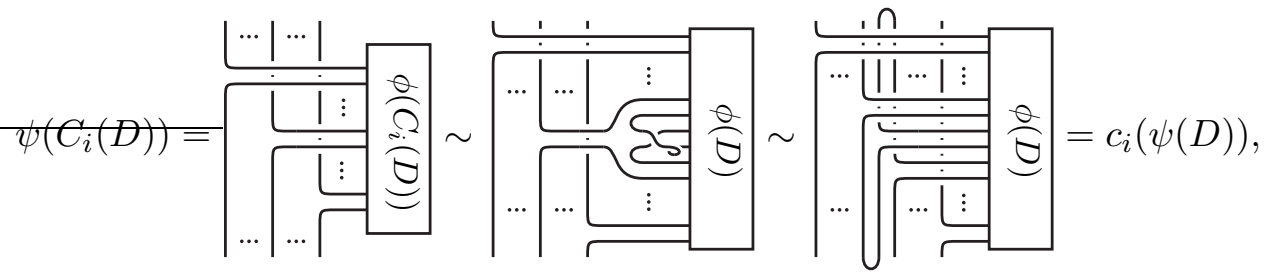

where we used that the $(2 i-1)$-th and $2 i$-th inputs of $\phi(D)$ are connected (since it is a ribbon handle).

Lemma 4.4 We have $c_{i} c_{j}=c_{j} c_{i+2}$ and $C_{i} C_{j}=C_{j} C_{i+2}$ for any $i \geq j$.

Proof This results directly from the definitions of the contraction operators.

\subsection{From ribbon string links to Hopf diagram}

In this section, we extend the monoidal functor $\Psi_{0}: \operatorname{RPB} \rightarrow \overline{\mathcal{D}} \boldsymbol{i a g}^{S}$ to a monoidal functor $\Psi: \mathrm{RSL} \rightarrow \overline{\mathcal{D}} \boldsymbol{i a g}^{S}$. The construction of this extension follows, broadly speaking, the same pattern as the proof of [2, Theorem 3]. The point is to see that a ribbon string link can be obtained from a ribbon pure braid by a sequence of contractions. This will at least show that $\Psi$ extends uniquely and suggest a construction for it. We then must check the coherence of this construction, that is, its independence from the choices we made.

The main trick we use consists in "pulling a max to the top line". Let $\Gamma$ be a tangle diagram with a local $\max m$, with $n$ outputs. We may write $\Gamma$ as in Figure 16(a) where $U, V$ are tangle diagrams. Let $i$ be an integer, $1 \leq i \leq n+1$. Let $j$ be the number of strands to the left of $m$ on the same horizontal line. Let $U \cup \ell$ be a tangle diagram obtained from $U$ by inserting a new component $\ell$ going from a point between the $j$-th and $(j+1)$-th inputs of $U$ to a point between the $(i-1)$-th and $i$-th outputs of $U$, see Figure 16(b) We assume also that $\ell$ has no local extremum. Let $U_{\ell}=\Delta_{\ell}(U \cup \ell)$ be the tangle diagram obtained from $U \cup \ell$ by doubling $\ell$. Set $\Gamma_{\ell}=U_{\ell} V$, see Figure 16(c). We say that $\Gamma_{\ell}$ is obtained from $\Gamma$ by pulling $m$ to the top in the $i$-th position (along the path $\ell$ ). Likewise, one defines the action of pulling a local min to the bottom.

We define $\Psi:$ RSL $\rightarrow \overline{\mathcal{D} i a g}^{S}$ as follows: on objects $n \in \mathbb{N}$, set $\Psi(n)=n$.

Let $n$ be a non-negative integer and $T$ be a ribbon $n$-string link. Consider 


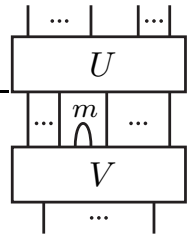

(a) $\Gamma$

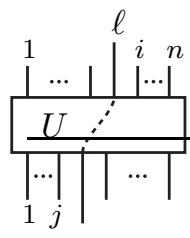

(b) $U \cup \ell$

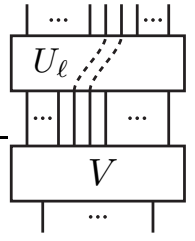

(c) $\Gamma_{\ell}$

Figure 16: Pulling a max to the top line

a diagram of $T$. For each local extremum pointing to the right (once the strands canonically oriented from bottom to top), modify the diagram using the following rule:

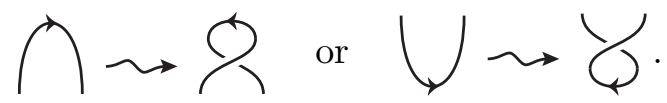

This leads to a diagram $\Gamma$ which is left handed, that is, with all local extrema pointing to the left. Pulling all local max to the top and all local min to the bottom, we obtain a diagram of a pure braid. Here is an algorithm. Denote by $m_{i}$ the number of local max (which is equal to the number of local min) on the $i$-th component of $\Gamma$. Let $m(\Gamma)=m_{1}+\cdots+m_{n}$ be the number of local max of $\Gamma$. If $m(\Gamma)=0$, we are already done. Otherwise, chose $i$ maximal so that $m_{i}>0$. Let $m$ be the first $\max$ and $m^{\prime}$ be the first min you meet on the $i$-th component, going from bottom to top. Pull $m$ to the top, in the $(i+1)$-th position, and $m^{\prime}$ to the bottom, in the $i$-th position. Let $\Gamma^{\prime}$ be the diagram so constructed. Then $\Gamma^{\prime}$ is a string link diagram, with $m\left(\Gamma^{\prime}\right)=m(\Gamma)-1$. Let us denote by $\{\Gamma\}$ the ribbon string link defined by the diagram $\Gamma$. Then $\{\Gamma\}=c_{i+1}\left\{\Gamma^{\prime}\right\}$, where $c_{i+1}$ is the $(i+1)$-th contraction as in Section 4.3, Repeating $m(\Gamma)$ times this transformation yields a pure braid diagram $P$ with $n+2 m(\Gamma)$ strands, and we have $\{\Gamma\}=c_{j_{m(\Gamma)}} \cdots c_{j_{1}}\{P\}$ where $1 \leq j_{1} \leq \cdots \leq j_{m(\Gamma)} \leq n$, and $j_{k}$ takes $m_{i}$ times the value $i+1$. Note that

$$
T=\left(t_{1}^{\alpha_{1}} \cdots t_{n}^{\alpha_{n}}\right) c_{j_{m(\Gamma)}} \cdots c_{j_{1}}\{P\}
$$

where $\alpha_{i}$ is the number of modifications (22) made on the $i$-th component and $t_{i} \in \mathrm{RPB}_{n}$ is as in Section 4.2. Finally, as suggested by Lemmas 4.2 and 4.3 . set:

$$
\Psi(T)=\left(\Omega_{1}^{\alpha_{1}} \cdots \Omega_{n}^{\alpha_{n}}\right) C_{j_{m(\Gamma)}} \cdots C_{j_{1}} \Psi_{0}(\{P\}),
$$

where $\Psi_{0}: \mathrm{RPB} \rightarrow \overline{\mathcal{D}} \boldsymbol{i a g}^{S}$ is the functor of Lemma 4.2, the $\Omega_{j}=\Psi_{0}\left(t_{j}\right)$ are the Hopf diagrams of Section 4.2 and the $C_{j}$ are the contractions on Hopf diagrams defined in Section 4.3 . 
A component of a ribbon string link is said to be trivial if it is trivial as an unframed long knot (up to isotopy). Note that each contraction $c_{j_{k}}$ in (23) is performed on a ribbon string link with trivial $j_{k}$-th component.

Theorem 4.5 The functor $\Psi: \mathrm{RSL} \rightarrow \overline{\mathcal{D}} \boldsymbol{i a g}^{S}$ is well-defined, monoidal, and satisfies $\bar{\psi} \circ \Psi=1_{\mathrm{RSL}}$. Moreover, $\Psi$ is the unique functor from RSL to $\overline{\mathcal{D} i a g} S$ satisfying:

(a) $\Psi(P)=\Psi_{0}(P)$ for any ribbon pure braid $P$;

(b) $\Psi\left(c_{i}(T)\right)=C_{i} \Psi(T)$ for any $1<i<n$ and any ribbon $n$-string link $T$ with trivial $i$-th component.

Remark in particular that $\Psi$ is injective and $\bar{\psi}$ (and so $\psi$ ) is surjective.

We prove the theorem in Section 4.6.

Set $\Phi=\Psi \circ G$ : RHand $\rightarrow \overline{\mathcal{D} i a g}^{S}$, where $G$ : RHand $\rightarrow$ RSL is the monoidal isomorphism defined in Section 2.3. From Proposition 2.1] and Theorem 4.5, we immediately deduce that:

Corollary 4.6 The functor $\Phi:$ RHand $\rightarrow \overline{\mathcal{D}}$ iag $^{S}$ is monoidal and satisfies $\bar{\phi} \circ \Phi=1_{\text {RHand }}$.

Note in particular that $\Phi$ is injective and that $\bar{\phi}$ (and so $\phi$ ) is surjective.

\subsection{Summary}

The previous results may be summarized in the commutativity of the following diagram:

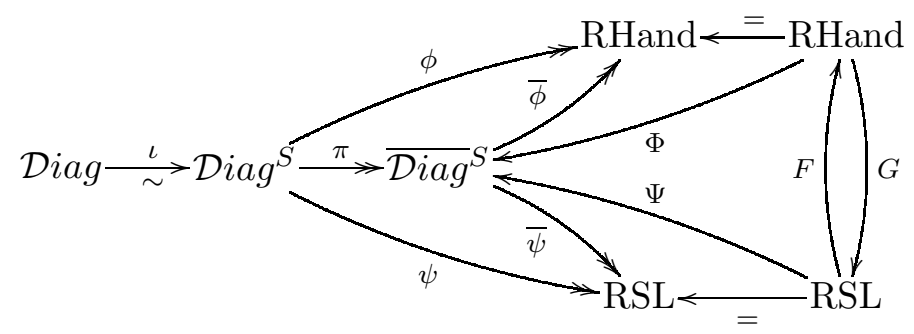

At this stage, we do not know whether the functors $\bar{\phi}$ and $\Phi$ (resp. $\bar{\psi}$ and $\Psi$ ) are isomorphisms and so inverse each other. 


\subsection{Proof of Theorem 4.5}

Before proving Theorem 4.5. we first establish some lemmas.

Lemma 4.7 Let $P$ be a ribbon pure $n$-braid and $1 \leq i \leq n+1$. Insert a new component $\ell$ between the $(i-1)$-th and $i$-th strand of $P$ so that $P \cup \ell$ is a ribbon pure $(n+1)$-braid. Let $P_{\ell}=\Delta_{\ell}(P \cup \ell)$ be the ribbon pure $(n+2)$-braid from $P \cup \ell$ by doubling $\ell$. Then:

(a) The equalities of Figure 17 hold;

(b) $C_{i}\left(\Psi_{0}\left(P_{\ell}\right) D\right)=\Psi_{0}(P) C_{i}(D)$ and $C_{i+1}\left(D \Psi_{0}\left(P_{\ell}\right)\right)=C_{i+1}(D) \Psi_{0}(P)$ for any Hopf diagram $D$ with $(n+2)$ inputs.

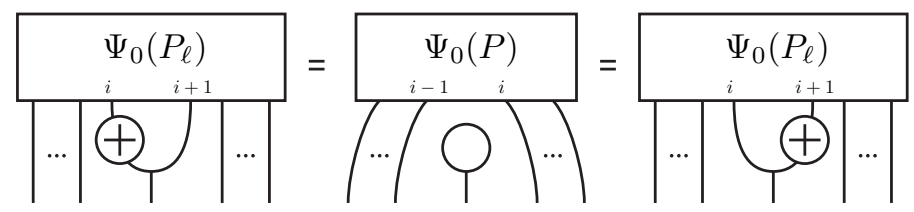

Figure 17

Proof Let us prove Part (a) by induction on the length $m$ of $P \cup l$ in the generators $\sigma_{k, l}^{ \pm 1}$ and $t_{k}^{ \pm 1}$ of $\mathrm{RPB}_{n+1}$. If $m=0$, then it is an immediate consequence of (2) and (5). Suppose that $m=1$. Given a ribbon pure braid $Q$, denote by $\Delta_{i}(Q)$ (resp. $\delta_{i}(Q)$ ) the ribbon pure braid obtained from $Q$ by doubling (resp. deleting) its $i$-th component. We have to verify that the statement is true for $P=\delta_{i}\left(\sigma_{k, l}^{ \pm 1}\right)$ and $P_{\ell}=\Delta_{i}\left(\sigma_{k, l}^{ \pm 1}\right)$, and for $P=\delta_{i}\left(t_{k}^{ \pm 1}\right)$ and $P_{\ell}=\Delta_{i}\left(t_{k}^{ \pm 1}\right)$. This can be done case by case by using the descriptions of Table 1. Examples of such verifications are depicted in Figure 18

\begin{tabular}{|c|c|c|}
\cline { 2 - 3 } \multicolumn{1}{c|}{} & $\Delta_{i}\left(\sigma_{k, l}^{ \pm 1}\right)$ & $\delta_{i}\left(\sigma_{k, l}^{ \pm 1}\right)$ \\
\hline$i<k$ & $\sigma_{k+1, l+1}^{ \pm 1}$ & $\sigma_{k-1, l-1}^{ \pm 1}$ \\
\hline$i=k$ & $\left(\sigma_{i, l+1} \sigma_{i+1, l+1}\right)^{ \pm 1}$ & $I_{n}$ \\
\hline$k<i<l$ & $\sigma_{k, l+1}^{ \pm 1}$ & $\sigma_{k, l-1}^{ \pm 1}$ \\
\hline$i=l$ & $\left(\sigma_{k, i} \sigma_{k, i+1}\right)^{ \pm 1}$ & $I_{n}$ \\
\hline$l<i$ & $\sigma_{k, l}^{ \pm 1}$ & $\sigma_{k, l}^{ \pm 1}$ \\
\hline
\end{tabular}

\begin{tabular}{|c|c|c|}
\cline { 2 - 3 } \multicolumn{1}{c|}{} & $\Delta_{i}\left(t_{k}^{ \pm 1}\right)$ & $\delta_{i}\left(t_{k}^{ \pm 1}\right)$ \\
\hline$i<k$ & $t_{k+1}^{ \pm 1}$ & $t_{k-1}^{ \pm 1}$ \\
\hline$i=k$ & $\sigma_{i, i+1}^{ \pm 1} t_{i}^{ \pm 1} t_{i+1}^{ \pm 1}$ & $I_{n}$ \\
\hline$k<i$ & $t_{k}^{ \pm 1}$ & $t_{k}^{ \pm 1}$ \\
\hline
\end{tabular}

Table 1 

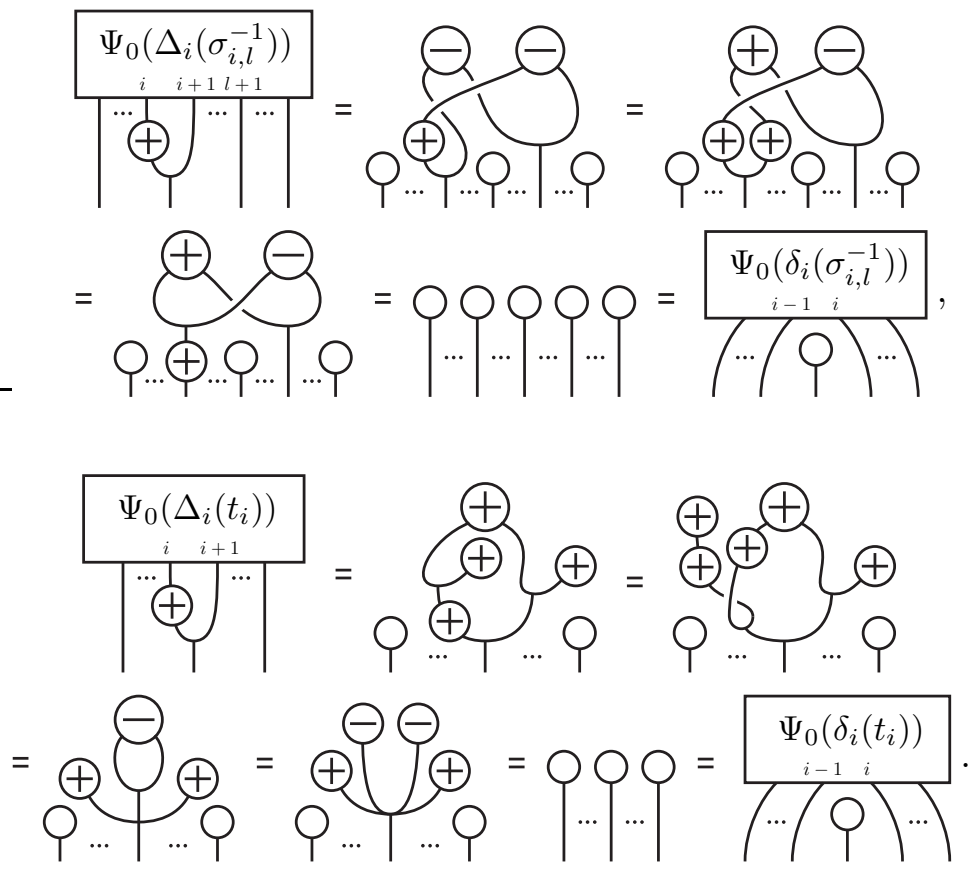

Figure 18

Let $m \geq 1$ and suppose the statement true for rank $m$. Let $P$ and $\ell$ such that $P \cup \ell=w_{1} \ldots w_{m+1}$ where the $w_{j}$ are generators of $\mathrm{RPB}_{n+1}$. Remark that $P_{\ell}=$ $\Delta_{i}\left(w_{1}\right) \Delta_{i}\left(w_{2} \cdots w_{m+1}\right)$ and $P=\delta_{i}\left(w_{1}\right) \delta_{i}\left(w_{2} \cdots w_{m+1}\right)$. By using (4), (2) , and the statement for ranks 1 and $m$, we get the equalities depicted in Figure 19. The left equality of Figure 17 is then true for $P$ and $\ell$. The right equality can be verified similarly by remarking that $P_{\ell}=\Delta_{i}\left(w_{1} \cdots w_{m}\right) \Delta_{i}\left(w_{m+1}\right)$. Hence the statement is true for rank $m+1$.

Let us prove Part (b). Let $D$ be a Hopf $(n+2)$-diagram. By using (11), (2), (4), and Part (a) of the lemma, we get the equalities of Figure 20, which mean that $C_{i}\left(\Psi_{0}\left(P_{\ell}\right) D\right)=\Psi_{0}(P) C_{i}(D)$. Likewise, one can show that $C_{i+1}\left(D \Psi_{0}\left(P_{\ell}\right)\right)=$ $C_{i+1}(D) \Psi_{0}(P)$.

Lemma 4.8 Let $n \geq 3$ and $1<i<n$ (resp. $0<i<n-1$ ). Let $P$ and $P^{\prime}$ be two pure $n$-braids which have diagrams which differ only inside a disk. Inside this disk, the $i$-th and $(i+1)$-th strands pass respectively to the front and the back of another strand. Suppose also that above (resp. below) the disk, the $i$-th and $(i+1)$-th strands run parallel, see Figure 21. Then:

$$
C_{i} \Psi_{0}(P)=C_{i} \Psi_{0}\left(P^{\prime}\right)\left(\text { resp. } C_{i+1} \Psi_{0}(P)=C_{i+1} \Psi_{0}\left(P^{\prime}\right)\right) .
$$



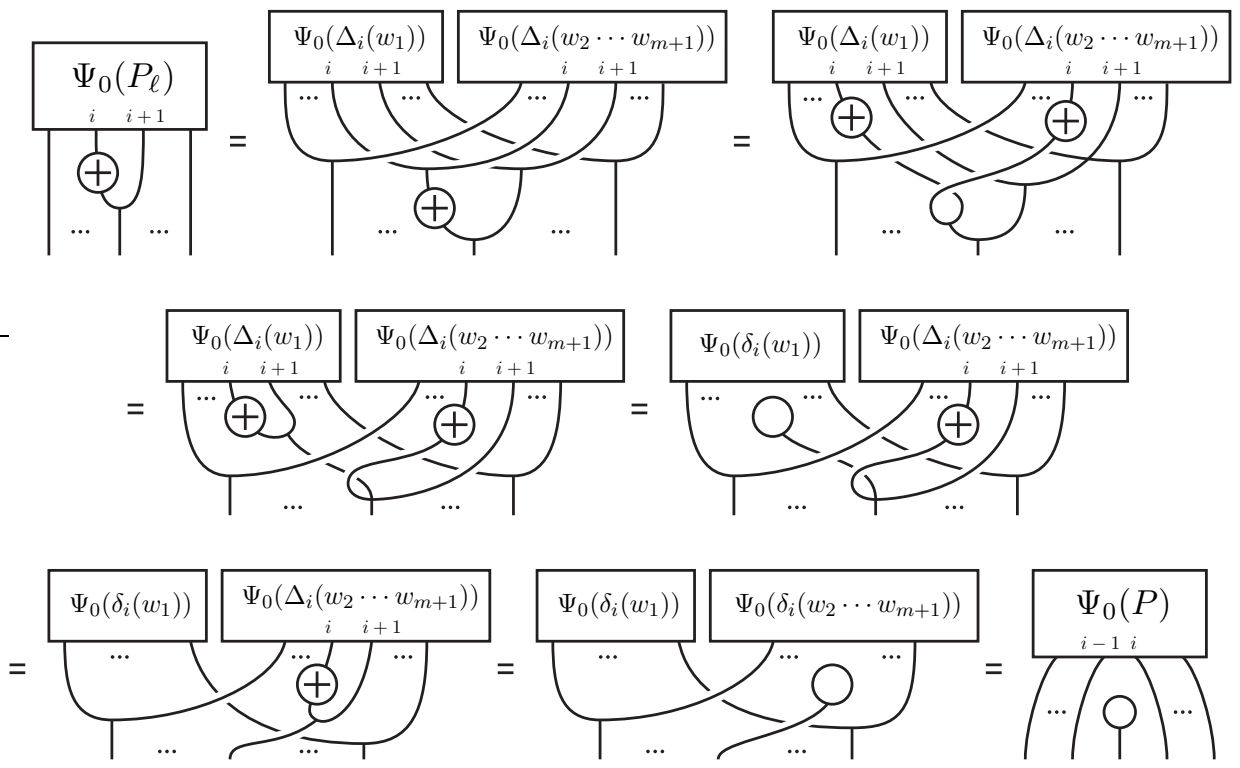

Figure 19
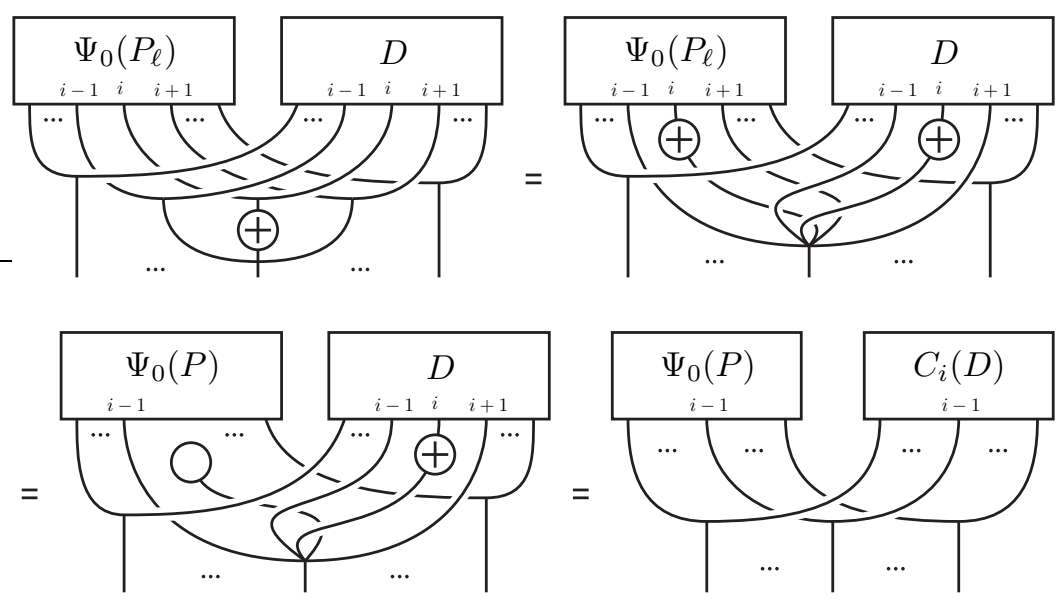

Figure 20

Proof Let us denote by $k$ the other component. Suppose that above the disk, the $i$-th and $(i+1)$-th strands run parallel (the resp. case can be done similarly). Assume first that $k<i$. We can write $P=A \sigma_{k, i} \sigma_{k, i+1} B$ and $P^{\prime}=A B$ where $A$ and $B$ are ribbon pure $n$-braids. Moreover $A=\Delta_{\ell}(E \cup \ell)$ where $E$ is ribbon 


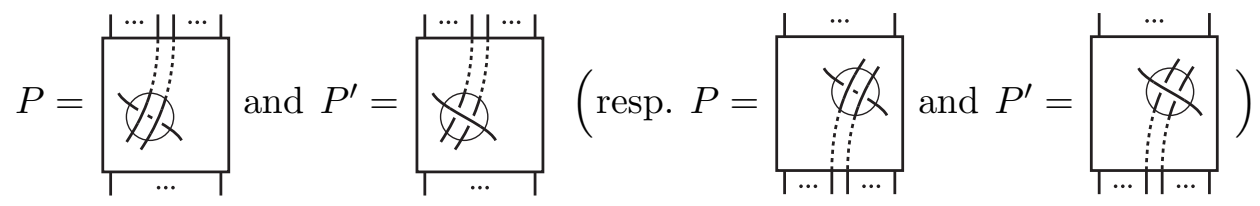

Figure 21

pure braid with $n-2$ strands and $\ell$ is a new component inserted in $E$ in $i$-th position. Now remark that $\sigma_{k, i} \sigma_{k, i+1}=\Delta_{\gamma}\left(I_{n-2} \cup \gamma\right)$ where $I_{n-2}$ is the trivial braid with $n-2$ strands and $\gamma$ is new component added to $I_{n-2}$ in $i$-th position so that $I_{n-2} \cup \gamma=\sigma_{k, i}$ in $\mathrm{RPB}_{n-1}$. Therefore, by using Lemma 4.7 we get:

$$
\begin{aligned}
C_{i} \Psi_{0}(P) & =C_{i} \Psi_{0}\left(A \sigma_{k, i} \sigma_{k, i+1} B\right)=C_{i} \Psi_{0}\left(\Delta_{\ell}(E \cup \ell) \Delta_{\gamma}\left(I_{n-2} \cup \gamma\right) B\right) \\
& =\Psi_{0}(E) \Psi_{0}\left(I_{n-2}\right) C_{i} \Psi_{0}(B)=\Psi_{0}(E) C_{i} \Psi_{0}(B) \\
& =C_{i} \Psi_{0}\left(\Delta_{\ell}(E \cup \ell) B\right)=C_{i} \Psi_{0}(A B)=C_{i} \Psi_{0}\left(P^{\prime}\right) .
\end{aligned}
$$

The case $k>i+1$ is done similarly by writing $P=A \sigma_{i, k} \sigma_{i+1, k} B$ and $P^{\prime}=$ $A B$.

Let us now prove Theorem 4.5. Let $n$ be a non-negative integer and $T$ be a ribbon $n$-string link. Consider a diagram $\Gamma_{T}$ of $T$. Applying rules (22) changes (in a unique manner) $\Gamma_{T}$ to a left handed diagram. Denote by $\alpha_{i}$ is the number of modifications (22) made on the $i$-th component of $\Gamma_{T}$. Then pulling maxima to the top and minima to the bottom as explained in Section 4.4 leads to a diagram $P$ of a ribbon pure braid so that $T=\left(t_{1}^{\alpha_{1}} \cdots t_{n}^{\alpha_{n}}\right) c_{j_{m}} \cdots c_{j_{1}}\{P\}$ for some $1 \leq$ $j_{1} \leq \cdots \leq j_{m} \leq n$. Pulling extrema in another way may lead to another diagram $P^{\prime}$ of a ribbon pure braid so that $T=\left(t_{1}^{\alpha_{1}} \cdots t_{n}^{\alpha_{n}}\right) c_{j_{m}} \cdots c_{j_{1}}\left\{P^{\prime}\right\}$. Now the ribbon pure braids $\{P\}$ and $\left\{P^{\prime}\right\}$ are related by moves described in Figure 21. Therefore, by using Lemmas 4.4 and 4.8 we obtain: $C_{j_{m}} \cdots C_{j_{1}} \Psi_{0}(\{P\})=$ $C_{j_{m}} \cdots C_{j_{1}} \Psi_{0}\left(\left\{P^{\prime}\right\}\right)$. Hence

$$
\Psi_{\Gamma_{T}}=\left(\Omega_{1}^{\alpha_{1}} \cdots \Omega_{n}^{\alpha_{n}}\right) C_{j_{m}} \cdots C_{j_{1}} \Psi_{0}(\{P\})
$$

only depends on the diagram $\Gamma_{T}$ of $T$.

Let us verify that $\Psi_{\Gamma_{T}}$ remains unchanged when applying to $\Gamma_{T}$ a Reidemeister's move, see Figure 5 Invariance under moves of type 2 or type 3 is a consequence of the existence of the functor $\Psi_{0}$. Invariance under moves of type 2 ' is a consequence of Lemmas 4.4 and 4.8

Suppose that a Reidemeister move of Type 0 is applied to $\Gamma_{T}$, and denote $\Gamma_{T}^{\prime}$ the diagram so obtained. There are four cases to consider, depending on the orientation of the considered strand and the direction (left or right handed) 


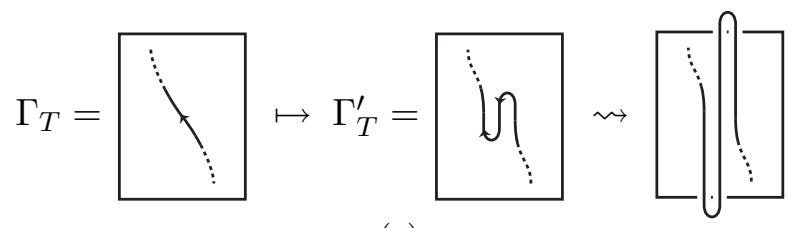

(a)

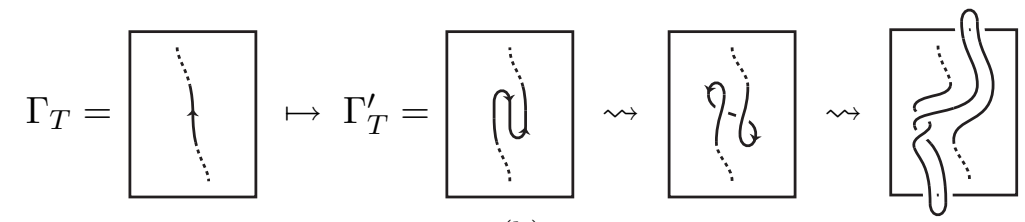

(b)

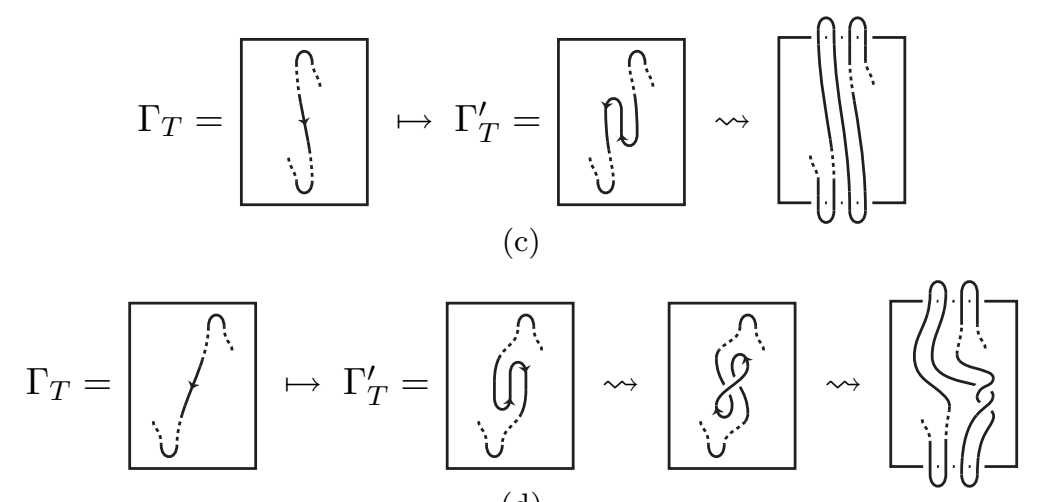

(d)

Figure 22

of the move. These cases, together with the way we apply the algorithm, are depicted in Figure 22. Let us for example verify invariance in the case depicted in Figure 22(d) Recall that applying the algorithm to $\Gamma_{T}$ gives rise to a diagram $P$ of a pure braid such that $\left\{\Gamma_{T}\right\}=\left(t_{1}^{\alpha_{1}} \cdots t_{n}^{\alpha_{n}}\right) c_{j_{m}} \cdots c_{j_{1}}\{P\}$. Let $i$ be the number of the component of $\Gamma_{T}$ on which the move is performed. Since the orientation of the strand is downwards and $T$ is a (canonically oriented) string link, we know that there exist a maximum just before and a minimum just after the place where the move is performed, when going through the $i$-th component from bottom to top (see the left picture of Figure 22(d). Let $c_{j_{r}}$ be the contraction corresponding to this pair of extrema when applying the algorithm to $\Gamma_{T}$ (we have $j_{r}=i+1$ ). Denote by $k$ the number of the component of $P$ where the move is performed. Up to using invariance under Reidemeister's moves of type 2 and type 3, we can write $\{P\}=U V$, with $U$ and $V$ pure braids, so that the move is performed on the $k$-th component between $U$ 


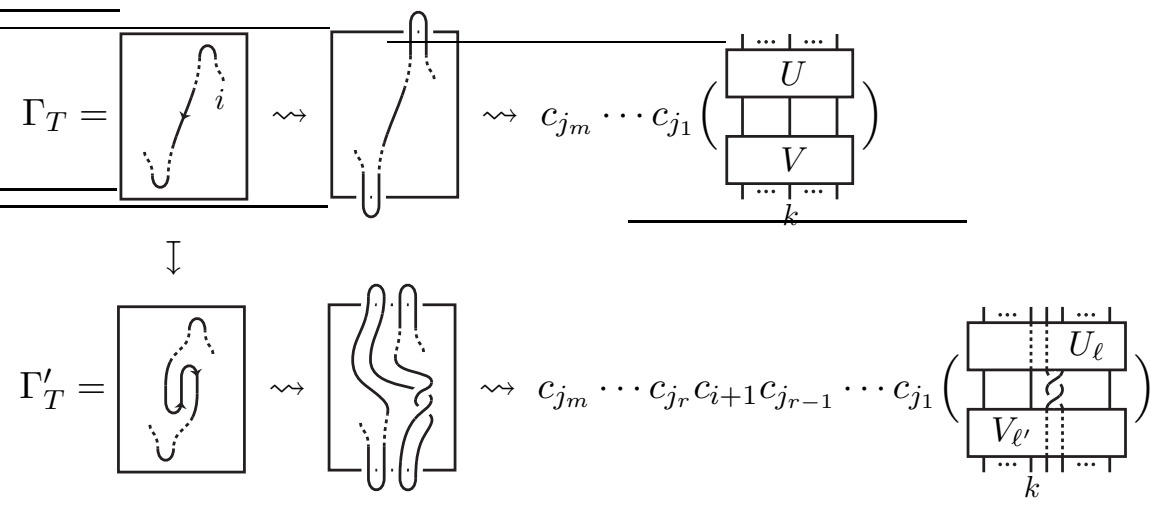

Figure 23

and $V$. Insert a new component $\ell$ in $U$ in $k$-th position and a new component $\ell^{\prime}$ in $V$ in $(k+1)$-th position. Set $U_{\ell}=\Delta_{\ell}(U \cup \ell)$ and $V_{\ell^{\prime}}=\Delta_{\ell^{\prime}}\left(V \cup \ell^{\prime}\right)$. As depicted in Figures 22(d) and 23, we can apply the algorithm to $\Gamma_{T}^{\prime}$ in such a way that:

$$
\left\{\Gamma_{T}^{\prime}\right\}=\left(t_{1}^{\alpha_{1}} \cdots t_{i}^{\alpha_{i}+2} \cdots t_{n}^{\alpha_{n}}\right) c_{j_{m}} \cdots c_{j_{r}} c_{i+1} c_{j_{r-1}} \cdots c_{j_{1}}\left(U_{\ell} \sigma_{k+1, k+2} V_{\ell^{\prime}}\right) .
$$

Now, by using (4), (6) and (9), we have, for any Hopf diagram $D$,

$$
C_{j}\left(\Omega_{p} D\right)= \begin{cases}\Omega_{p} C_{j}(D) & \text { for } p<j \\ \Omega_{j-1} C_{j}(D) & \text { for } p=j \\ \Omega_{p-2} C_{j}(D) & \text { for } p>j\end{cases}
$$

Moreover we have the equalities of Figure 24 where are used in particular (17), (9), (12) and Lemma 4.7(a). Then we get that:

$$
\begin{aligned}
C_{k} C_{k} \Psi_{0}\left(U_{\ell} \sigma_{k+1, k+2} V_{\ell^{\prime}}\right) & =C_{k} C_{k+2}\left(\Psi_{0}\left(U_{\ell}\right) \Sigma_{k+1, k+2} \Psi_{0}\left(V_{\ell^{\prime}}\right)\right) \\
& =\Omega_{k-1}^{-2} C_{k}\left(\Psi_{0}(U) \Psi_{0}(V)\right) \\
& =\Omega_{k-1}^{-2} C_{k} \Psi_{0}(\{P\}) .
\end{aligned}
$$

Therefore we can conclude that:

$$
\begin{aligned}
\psi_{\Gamma_{T}^{\prime}} & =\left(\Omega_{1}^{\alpha_{1}} \cdots \Omega_{i}^{\alpha_{i}+2} \cdots \Omega_{n}^{\alpha_{n}}\right) C_{j_{m}} \cdots C_{j_{r}} C_{i+1} C_{j_{r-1}} \cdots C_{j_{1}} \Psi_{0}\left(U_{\ell} \sigma_{k+1, k+2} V_{\ell^{\prime}}\right) \\
& =\left(\Omega_{1}^{\alpha_{1}} \cdots \Omega_{i}^{\alpha_{i}+2} \cdots \Omega_{n}^{\alpha_{n}}\right) C_{j_{m}} \cdots \widehat{C_{j_{r}}} \cdots C_{j_{1}} C_{k} C_{k} \Psi_{0}\left(U_{\ell} \sigma_{k+1, k+2} V_{\ell^{\prime}}\right) \\
& =\left(\Omega_{1}^{\alpha_{1}} \cdots \Omega_{i}^{\alpha_{i}+2} \cdots \Omega_{n}^{\alpha_{n}}\right) C_{j_{m}} \cdots \widehat{C_{j_{r}}} \cdots C_{j_{1}}\left(\Omega_{k-1}^{-2} C_{k} \Psi_{0}(\{P\})\right) \\
& =\left(\Omega_{1}^{\alpha_{1}} \cdots \Omega_{i}^{\alpha_{i}+2} \cdots \Omega_{n}^{\alpha_{n}}\right) \Omega_{i}^{-2} C_{j_{m}} \cdots \widehat{C_{j_{r}}} \cdots C_{j_{1}} C_{k} \Psi_{0}(\{P\}) \quad \text { by (24) } \\
& =\left(\Omega_{1}^{\alpha_{1}} \cdots \Omega_{n}^{\alpha_{n}}\right) C_{j_{m}} \cdots C_{j_{1}} \Psi_{0}(\{P\})=\psi_{\Gamma_{T}} .
\end{aligned}
$$



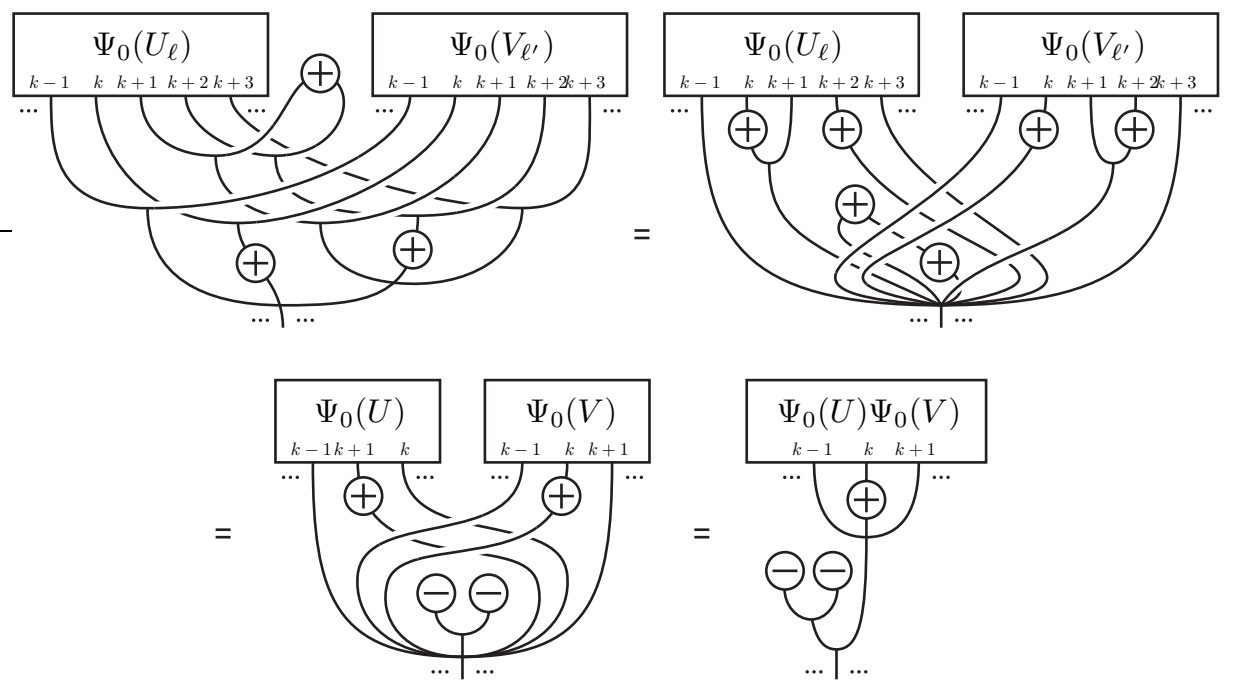

Figure 24

Invariance in the cases depicted in Figures 22(a) 22(b) and 22(c) can be checked as above.

By using similar techniques, one can show the invariance of $\Psi_{\Gamma_{T}}$ under Reidemeister's moves of type 1'. Hence we conclude that $\Psi_{\Gamma_{T}}$ remains unchanged when applying to $\Gamma_{T}$ a Reidemeister's move, and so $\Psi(T)=\Psi_{\Gamma_{T}}$ is well-defined.

We get directly from its construction that the functor $\Psi$ is monoidal and satisfies Condition (a). Let us check that it satisfies Condition (b). Let $1<i<n$ and $T$ be a ribbon $n$-string link with trivial $i$-th component. By applying the algorithm we have $T=\left(t_{1}^{\alpha_{1}} \cdots t_{n}^{\alpha_{n}}\right) c_{j_{m}} \cdots c_{j_{1}}(P)$ for some $1 \leq j_{1} \leq \cdots \leq j_{m} \leq n$ and some pure braid $P$. Since the $i$-th component of $T$ is trivial, we can apply the algorithm to a diagram of $c_{i}(T)$ in such a way that:

$c_{i}(T)=\left(t_{1}^{\alpha_{1}} \cdots t_{i-2}^{\alpha_{i-2}} t_{i-1}^{\alpha_{i-1}+\alpha_{i}+\alpha_{i+1}} t_{i}^{\alpha_{i+2}} \cdots t_{n-2}^{\alpha_{n}}\right) c_{j_{m}-2} \cdots c_{j_{k+1}-2} c_{i} c_{j_{k}} \cdots c_{j_{1}}(P)$, where $k$ is such that $j_{k+1}>i+1 \geq j_{k}$. Hence:

$$
\begin{aligned}
C_{i}(\Psi( & (T))=C_{i}\left(\Omega_{1}^{\alpha_{1}} \cdots \Omega_{n}^{\alpha_{n}} C_{j_{m}} \cdots C_{j_{1}} \Psi_{0}(P)\right) \\
& =\left(\Omega_{1}^{\alpha_{1}} \cdots \Omega_{i-1}^{\alpha_{i-1}+\alpha_{i}+\alpha_{i+1}} \cdots \Omega_{n-2}^{\alpha_{n}}\right) C_{i} C_{j_{m}} \cdots C_{j_{1}} \Psi_{0}(P) \text { by (24) } \\
& =\left(\Omega_{1}^{\alpha_{1}} \cdots \Omega_{i-1}^{\alpha_{i-1}+\alpha_{i}+\alpha_{i+1}} \cdots \Omega_{n-2}^{\alpha_{n}}\right) C_{j_{m}-2} \cdots C_{j_{k+1}-2} C_{i} C_{j_{k}} \cdots C_{j_{1}} \Psi_{0}(P) \\
& =\Psi\left(c_{i}(T)\right) .
\end{aligned}
$$

Uniqueness of a functor $\overline{\mathcal{D} i a g}^{S} \rightarrow$ RSL satisfying (a) and (b) comes from the fact that every ribbon string link can be realized as a sequence of contractions 
of a ribbon pure braid such that each of contraction is performed on a trivial component (see the algorithm described in Section 4.4).

Finally, let $T$ be a ribbon string link. We can always write $T=c_{j_{m}} \cdots c_{j_{1}}(P)$ for some ribbon pure braid $P$. Then we have:

$$
\begin{aligned}
\bar{\psi} \Psi(T) & =\bar{\psi} \Psi\left(c_{j_{m}} \cdots c_{j_{1}}(P)\right) \\
& =\bar{\psi}\left(C_{j_{m}} \cdots C_{j_{1}} \Psi_{0}(P)\right) \quad \text { by Conditions (a) and (b) } \\
& =c_{j_{m}} \cdots c_{j_{1}}\left(\bar{\psi} \Psi_{0}(P)\right) \quad \text { by Lemma } 4.3 \\
& =c_{j_{m}} \cdots c_{j_{1}}(P)=T \text { by Lemma } 4.2
\end{aligned}
$$

Hence $\bar{\psi} \circ \Psi=1_{\mathrm{RSL}}$. This completes the proof of Theorem 4.5

\section{Quantum invariants via Hopf diagrams and Kirby elements}

A general method is given in 14 for defining quantum invariants of 3-manifolds starting from a ribbon category (or a ribbon Hopf algebra). In this section, we explain the role played by Hopf diagrams in this theory. Note that this was the initial motivation of this work.

\subsection{Dinatural transformations and coends}

We give here definitions adapted to our purposes. For more general situations, we refer to [8].

Let $\mathcal{C}$ be a category with left duals. By a dinatural transformation of $\mathcal{C}$, we shall mean a pair $(Z, d)$ consisting in an object $Z$ of $\mathcal{C}$ and a family $d$, indexed by $\mathrm{Ob}(\mathcal{C})$, of morphisms $d_{X}:{ }^{\vee} X \otimes X \rightarrow Z$ in $\mathcal{C}$ satisfying $d_{Y}\left(\operatorname{id} \vee_{Y} \otimes f\right)=$ $d_{X}\left({ }^{\vee} f \otimes \operatorname{id}_{X}\right)$ for any morphism $f: X \rightarrow Y$.

By a coend of $\mathcal{C}$, we shall mean a dinatural transformation $(A, i)$ which is universal in the sense that, if $(Z, d)$ is any dinatural transformation, then there exists a unique morphism $r: A \rightarrow Z$ such that $d_{X}=r \circ i_{X}$ for all object $X$ in $\mathcal{C}$, see Figure 25. Note that a coend, if it exists, is unique (up to unique isomorphism).

Remarks (1) In general, the coend of $\mathcal{C}$ always exists in a completion of $\mathcal{C}$, namely the category of Ind-objects of $\mathcal{C}$ (see [6]). However, for simplicity, we will restrict to the case where the coend exists in $\mathcal{C}$. 


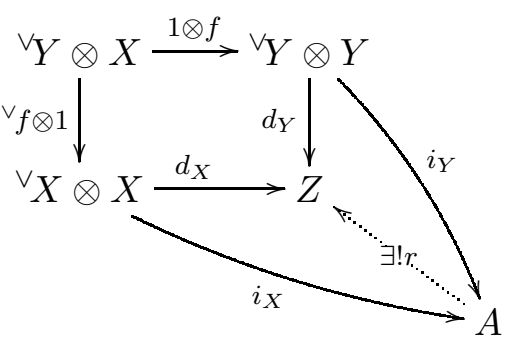

Figure 25

(2) If $\mathcal{C}$ is the category of representations of a finite-dimensional Hopf algebra or is a premodular category, then the coend exists in $\mathcal{C}$, see [14].

Assume that $(A, i)$ is the coend of $\mathcal{C}$. Let the morphisms ev ${ }_{X}{ }^{\vee} X \otimes X \rightarrow \mathbf{1}$ and $\operatorname{coev}_{X}: \mathbf{1} \rightarrow X \otimes{ }^{\vee} X$ be the evaluation and coevaluation associated to the left dual ${ }^{\vee} X$ of an object $X$. The following dinatural transformations:

$$
\left(i_{X} \otimes i_{X}\right)\left(1 \otimes \operatorname{coev}_{X} \otimes 1\right):{ }^{\vee} X \otimes X \rightarrow A \otimes A \text { and } \quad \mathrm{ev}_{X}:{ }^{\vee} X \otimes X \rightarrow \mathbf{1}
$$

factorize respectively to morphisms $\Delta_{A}: A \rightarrow A \otimes A$ (the coproduct) and $\varepsilon_{A}: A \rightarrow \mathbf{1}$ (the counit). This makes $A$ a coalgebra in the category $\mathcal{C}$.

\subsection{The coend of a ribbon category}

Let $\mathcal{C}$ be a ribbon category (see [13]) and assume that the coend $(A, i)$ of $\mathcal{C}$ exists. We denote the braiding of $\mathcal{C}$ by $c_{X, Y}: X \otimes Y \rightarrow Y \otimes X$, and the twist of $\mathcal{C}$ by $\theta_{X}: X \rightarrow X$.

The coalgebra structure $\left(\Delta_{A}, \varepsilon_{A}\right)$ on the object $A$ (see Section 5.1) extends to a structure of a Hopf algebra, see 6 . This means that there exist morphisms $\mu_{A}: A \otimes A \rightarrow A$ (the product), $\eta_{A}: \mathbf{1} \rightarrow A$ (the unit), and $S_{A}: A \rightarrow A$ (the antipode). They satisfy the same axioms as those of a Hopf algebra except the usual flip is replaced by the braiding $c_{A, A}: A \otimes A \rightarrow A \otimes A$. Namely, the unit morphism is $\eta_{A}=i_{\mathbf{1}}: \mathbf{1}=\mathrm{V}^{\mathbf{1}} \otimes \mathbf{1} \rightarrow A$. By using the universal property of a coend, the product $\mu_{A}$ and the antipode $S_{A}$ are defined as follows:

$$
\begin{aligned}
& \mu_{A}\left(i_{X} \otimes i_{Y}\right)=i_{Y \otimes X}\left(1 \vee_{X} \otimes c_{X},_{Y}{ } \otimes Y\right):{ }^{\vee} X \otimes X \otimes{ }^{\vee} Y \otimes Y \rightarrow A,
\end{aligned}
$$

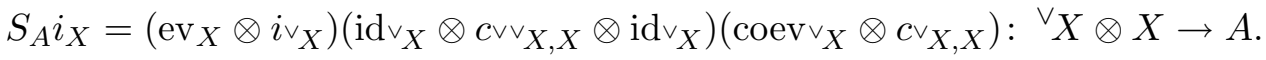

\footnotetext{
${ }^{1}$ For the product $\mu_{A}$, use it twice.
} 
Note that $S_{A}$ is invertible, with inverse $S_{A}^{-1}: A \rightarrow A$ defined via:

$$
S_{A}^{-1} i_{X}=\left(\mathrm{ev}_{X} \otimes i^{\vee_{X}}\right)\left(\mathrm{id}_{\vee_{X}} \otimes c_{\vee^{\vee}{ }_{X, X}}^{-1} \otimes \operatorname{id} \vee_{X}\right)\left(\operatorname{coev}^{\vee_{X}} \otimes c_{\vee_{X}, X}^{-1}\right):{ }^{\vee} X \otimes X \rightarrow A .
$$

Let us define the morphisms $\omega_{A}: A \otimes A \rightarrow \mathbf{1}$ and $\theta_{A}^{ \pm}: A \rightarrow \mathbf{1}$ as follows:

$$
\begin{aligned}
& \theta_{A}^{ \pm} i_{X}=\operatorname{ev}_{X}\left(\operatorname{id}_{X} \otimes \theta_{X}^{ \pm 1}\right):{ }^{\vee} X \otimes X \rightarrow \mathbf{1}, \\
& \omega_{A}\left(i_{X} \otimes i_{Y}\right)=\omega_{X, Y}:{ }^{\vee} X \otimes X \otimes{ }^{\vee} Y \otimes Y \rightarrow \mathbf{1},
\end{aligned}
$$

where $\omega_{X, Y}=\left(\operatorname{ev}_{X} \otimes \mathrm{ev}_{Y}\right)\left(\operatorname{idv}_{X} \otimes c v_{Y, X} c_{X, \vee_{Y}} \otimes i v_{Y}\right)$. It can be shown that $\omega_{A}$ is a Hopf pairing, see [14. Finally, we set:

$$
\omega_{A}^{+}=\omega_{A}\left(S_{A}^{-1} \otimes \operatorname{id}_{A}\right) \quad \text { and } \quad \omega_{A}^{-}=\omega_{A} .
$$

\subsection{Hopf diagrams and factorization}

Let $\mathcal{C}$ be a ribbon category. Assume that the coend $(A, i)$ of $\mathcal{C}$ exists. Consider the Hopf algebra structure of $A$ and the morphisms $\omega_{A}^{ \pm}$and $\theta_{A}^{ \pm}$as in Section 5.2 .

Let $T$ be a ribbon $n$-handle. Recall that for any objects $X_{1}, \ldots, X_{n}$ of $\mathcal{C}$, the handle $T$ defines a morphism $T_{X_{1}, \ldots, X_{n}}:{ }^{\vee} X_{1} \otimes X_{1} \otimes \cdots \otimes{ }^{\vee} X_{n} \otimes X_{n} \rightarrow \mathbf{1}$ by canonically orienting $T$ (see Section 2.2) and decorating the $k^{\text {th }}$ component of $T$ by $X_{k}$. Hence it can be factorized thought the coend to a morphism $T_{\mathcal{C}}: A^{\otimes n} \rightarrow \mathbf{1}$ so that:

$$
T_{X_{1}, \ldots, X_{n}}=T_{\mathcal{C}} \circ\left(i_{X_{1}} \otimes \cdots \otimes i_{X_{n}}\right)
$$

for all objects $X_{1}, \ldots, X_{n}$ of $\mathcal{C}$.

Let $\overline{\mathcal{D}}^{S}$ be the braided category defined in Section 4.1

Theorem 5.1 (a) There exists a unique braided functor $e_{\mathcal{C}}: \overline{\mathcal{D}}^{S} \rightarrow \mathcal{C}$ sending the object $*$ to $A$ and the morphisms $\Delta, \varepsilon, S^{ \pm 1}, \omega_{ \pm}, \theta_{ \pm}$to the morphims $\Delta_{A}, \varepsilon_{A}, S_{A}^{ \pm 1}, \omega_{A}^{ \pm}, \theta_{A}^{ \pm}$respectively.

(b) The functor $e_{\mathcal{C}}$ induces a monoidal functor $E_{\mathcal{C}}: \overline{\mathcal{D}_{i a g}} S \rightarrow \operatorname{Conv} \mathcal{C}(A, \mathbf{1})$.

(c) For any ribbon $n$-handle $T$, the factorization morphism $T_{\mathcal{C}}: A^{\otimes n} \rightarrow \mathbf{1}$ defined by $T$ is given by $T_{\mathcal{C}}=E_{\mathcal{C}}(D)$ where $D$ is any Hopf diagram such that $\bar{\phi}(D)=T$. In particular, $T_{\mathcal{C}}=E_{\mathcal{C}} \circ \Phi(T)$.

Remark 5.2 We expect to generalize this result to categories $\mathcal{C}$ which are not ribbon but only turban, see [2]. 
Proof Let us prove Part (a). Uniqueness of the functor $e_{\mathcal{C}}$ is clear since we impose the image of all its generators. Its existence comes from the fact that the relations imposed on the generators $\Delta, \varepsilon, S^{ \pm 1}, \omega_{ \pm}, \theta_{ \pm}$and $\tau_{*, *}^{ \pm 1}$ in $\overline{\mathcal{D}}^{S}$ are still true in $\mathcal{C}$ when replacing them by $\Delta_{A}, \varepsilon_{A}, S_{A}^{ \pm 1}, \omega_{A}^{ \pm}, \theta_{A}^{ \pm}$and $c_{A, A}^{ \pm 1}$ respectively (since $\omega_{A}$ is a Hopf pairing and $\theta_{A}$ is defined using the twist of $\mathcal{C}$ ).

Part (b) is a direct consequence of $\overline{\mathcal{D}} \boldsymbol{i a g}^{S}=\operatorname{Conv}_{\overline{\mathcal{D}}^{S}}(*, \mathbf{1})$.

Finally, let us prove Part (c). By the uniqueness of the factorization morphism via a coend, we have to show that if $D$ is a Hopf diagram with $n$ inputs, then

$$
\bar{\phi}(D)_{X_{1}, \ldots, X_{n}}=E_{\mathcal{C}}(D) \circ\left(i_{X_{1}} \otimes \cdots \otimes i_{X_{n}}\right)
$$

for any objects $X_{1}, \ldots, X_{n}$ of $\mathcal{C}$. Clearly, if (26) is true for two Hopf diagrams $D$ and $D^{\prime}$, then it is also true for the Hopf diagram $D \otimes D^{\prime}$.

Moreover, by definition of $\phi$ (see Figure 8) and of $\varepsilon_{A}, \omega_{A}^{ \pm}$and $\theta_{A}^{ \pm}$, (26) is true for the trivial Hopf diagram $\varepsilon \otimes \cdots \otimes \varepsilon$ and for the Hopf diagrams $\Sigma_{i, j}^{ \pm 1}$ and $\Omega_{k}^{ \pm 1}$ depicted in (21). Now suppose that (26) is true for some Hopf diagram $D$. Then, by definition of $\phi$ (see Figure 8) and of $\Delta_{A}, S_{A}^{ \pm 1}$ and $c_{A, A}^{ \pm 1}$, (26) remains true for the following diagrams:

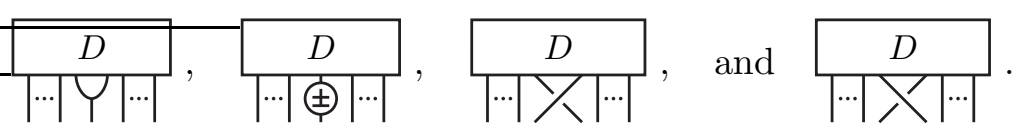

Hence we can deduce that (26) is always true.

\subsection{Kirby elements}

Let $\mathcal{C}$ and $A$ be as in Section 5.3 Let $L$ be a framed link in $S^{3}$ with $n$ components. Choose an orientation for $L$. There always exists a ribbon $n$-handle $T$ (not necessarily unique) such that $L$ is isotopic $T \circ(\cup \otimes \cdots \otimes \cup)$, where $\cup$ denotes the cup with counterclockwise orientation and $T$ is canonically oriented. For $\alpha \in \operatorname{Hom}_{\mathcal{C}}(\mathbf{1}, A)$, set:

$$
\tau_{\mathcal{C}}(L ; \alpha)=T_{\mathcal{C}} \circ \alpha^{\otimes n} \in \operatorname{End}_{\mathcal{C}}(\mathbf{1}),
$$

where $T_{\mathcal{C}}: A^{\otimes n} \rightarrow \mathbf{1}$ is defined as in (25). Following [14], by a Kirby element of $\mathcal{C}$ we shall mean a morphism $\alpha \in \operatorname{Hom}_{\mathcal{C}}(\mathbf{1}, A)$ such that, for any framed link $L, \tau_{\mathcal{C}}(L ; \alpha)$ is well-defined and invariant under isotopies and 2-handle slides of $L$.

In general, determining the set of the morphisms $T_{\mathcal{C}}$ when $T$ runs over ribbon handles is quite difficult. Nevertheless, by Theorem 5.1 the $T_{\mathcal{C}}$ 's belong to the set of morphisms given by evaluations of Hopf diagrams. Hence a criterion for a morphism to be a Kirby element: 
Corollary 5.3 Let $\alpha: \mathbf{1} \rightarrow A$ in $\mathcal{C}$. Suppose that the two following conditions are satisfied:

(a) for any integer $n \geq 1$ and any Hopf diagram $D$ with $n$ entries, we have:

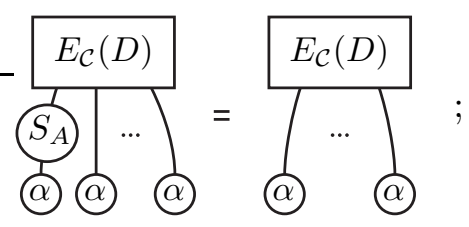

(b) for any integer $n \geq 2$ and any Hopf diagram $D$ with $n$ entries, we have:

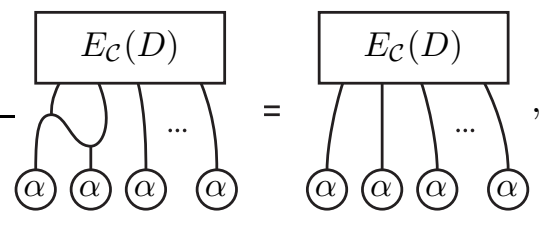

where $h$ and $Y$ denote $\mu_{A}: A \otimes A \rightarrow A$ and $\Delta_{A}: A \rightarrow A \otimes A$ respectively.

Then $\alpha$ is a Kirby element of $\mathcal{C}$.

Remark 5.4 One recovers the fact (see [14, Theorem 2.5]) that a morphism $\alpha: \mathbf{1} \rightarrow A$ is a Kirby element if it satisfies:

$$
\left\{\begin{array}{l}
S_{A} \alpha=\alpha, \\
\left(\mu_{A} \otimes \operatorname{id}_{A}\right)\left(\operatorname{id}_{A} \otimes \Delta_{A}\right)(\alpha \otimes \alpha)=\alpha \otimes \alpha,
\end{array}\right.
$$

or, in case $\mathcal{C}$ is linear, if the morphisms

$$
\left\{\begin{array}{l}
S_{A} \alpha-\alpha \\
\left(\mu_{A} \otimes \operatorname{id}_{A}\right)\left(\operatorname{id}_{A} \otimes \Delta_{A}\right)(\alpha \otimes \alpha)-\alpha \otimes \alpha,
\end{array}\right.
$$

are negligible.

Proof We adapt the proof of [14, Theorem 2.5] to this more general situation. Let $L=L_{1} \cup \cdots \cup L_{n}$ be a framed link. Choose an orientation of $L$ and a ribbon $n$-handle $T$ such that $L$ is isotopic $T \circ(\cup \otimes \cdots \otimes \cup)$.

Suppose firstly that we reverse the orientation of $L_{i}$. Let us denote by $L^{\prime}$ the oriented framed link obtained from $L$ by this orientation change. As in the proof of [14, Theorem 2.5], we can choose a ribbon $n$-handle $T^{\prime}$ such $L^{\prime}$ is isotopic to $T^{\prime} \circ(\cup \otimes \cdots \otimes \cup)$ and $T_{\mathcal{C}}^{\prime}=T_{\mathcal{C}} \circ\left(\operatorname{id}_{A^{\otimes(i-1)}} \otimes S_{A} \otimes \operatorname{id}_{A^{\otimes(n-i)}}\right)$. Now 
let $D$ be a Hopf diagram such that $\bar{\phi}(D)=T$. Then $T_{\mathcal{C}}=E_{\mathcal{C}}(D)$ and so

$$
\begin{aligned}
\tau_{\mathcal{C}}\left(L^{\prime} ; \alpha\right) & =E_{\mathcal{C}}(D)\left(\mathrm{id}_{A^{\otimes(i-1)}} \otimes S_{A} \otimes \mathrm{id}_{A^{\otimes(n-i)}}\right) \alpha^{\otimes n} \\
& =E_{\mathcal{C}}(D)\left(c_{A, A^{\otimes(i-1)}} \otimes \mathrm{id}_{A^{(n-i)}}\right)\left(S_{A} \alpha \otimes \alpha^{\otimes n-1}\right) \\
& =E_{\mathcal{C}}\left(D^{\prime}\right)\left(S_{A} \alpha \otimes \alpha^{\otimes n-1}\right) \\
& =E_{\mathcal{C}}\left(D^{\prime}\right) \alpha^{\otimes n} \quad \text { by Condition (a) } \\
& =E_{\mathcal{C}}(D)\left(c_{A, A^{\otimes(i-1)}} \otimes \mathrm{id}_{A^{(n-i)}}\right) \alpha^{\otimes n} \\
& =E_{\mathcal{C}}(D) \alpha^{\otimes n}=\tau_{\mathcal{C}}(L ; \alpha),
\end{aligned}
$$

where

$$
D^{\prime}=\frac{D_{i}}{\mid \cdots /}|\cdots| .
$$

Hence $\tau_{\mathcal{C}}(L ; \alpha)$ does not depend on the choice of an orientation for $L$.

Suppose now that the component $L_{2}$ slides over the component $L_{1}$. Let $L_{1}^{\prime}$ be a parallel copy of $L_{1}$ and set $L^{\prime}=L_{1} \cup\left(L_{1}^{\prime} \# L_{2}\right) \cup L_{3} \cup \cdots \cup L_{n}$. As in the proof of 14. Theorem 2.5], we can choose a ribbon $n$-handle $T^{\prime}$ such that $L^{\prime}$ is isotopic to $T^{\prime} \circ(\cup \otimes \cdots \otimes \cup)$ and $T_{\mathcal{C}}^{\prime}=T_{\mathcal{C}} \circ\left(\left(\mu_{A} \otimes \mathrm{id}_{A}\right)\left(\mathrm{id}_{A} \otimes \Delta_{A}\right) \otimes \mathrm{id}_{A \otimes(n-2)}\right)$. Hence, choosing a Hopf diagram $D$ such that $\bar{\phi}(D)=T$, we get that $T_{\mathcal{C}}=E_{\mathcal{C}}(D)$ and so

$$
\begin{aligned}
\tau_{\mathcal{C}}\left(L^{\prime} ; \alpha\right) & =E_{\mathcal{C}}(D)\left(\left(\mu_{A} \otimes \operatorname{id}_{A}\right)\left(\operatorname{id}_{A} \otimes \Delta_{A}\right) \otimes \operatorname{id}_{A \otimes(n-2)}\right) \alpha^{\otimes n} \\
& =E_{\mathcal{C}}(D) \alpha^{\otimes n} \quad \text { by Condition }(\mathrm{b}) \\
& =\tau_{\mathcal{C}}(L ; \alpha) .
\end{aligned}
$$

Likewise, using the braiding, we can show that $\tau_{\mathcal{C}}(L ; \alpha)$ is invariant under the other handle slides. Hence $\alpha$ is a Kirby element of $\mathcal{C}$.

\subsection{Quantum invariants of 3-manifolds}

Recall (see [5]) that every closed, connected and oriented 3-dimensional manifold can be obtained from $S^{3}$ by surgery along a framed link $L \subset S^{3}$.

For any framed link $L$ in $S^{3}$, we will denote by $S_{L}^{3}$ the 3 -manifold obtained from $S^{3}$ by surgery along $L$, by $n_{L}$ the number of components of $L$, and by $b_{-}(L)$ the number of negative eigenvalues of the linking matrix of $L$.

A Kirby element $\alpha$ of $\mathcal{C}$ is said to be normalizable if $\theta_{A}^{+} \alpha$ and $\theta_{A}^{-} \alpha$ are invertible in the semigroup $\operatorname{End}_{\mathcal{C}}(\mathbf{1})$. To each normalizable Kirby element $\alpha$ is associated an invariant $\tau_{\mathcal{C}}(M ; \alpha)$ of (closed, connected, and oriented) 3-manifolds $M$ with values in $\operatorname{End}_{\mathcal{C}}(\mathbf{1})$, see [14, Proposition 2.3]. From Theorem 5.1] we immediately deduce that: 
Corollary 5.5 For a normalizable Kirby element $\alpha$ and any framed link $L$, we have

$$
\tau_{\mathcal{C}}\left(S_{L}^{3} ; \alpha\right)=\left(\theta_{A}^{+} \alpha\right)^{b_{-}(L)-n_{L}}\left(\theta_{A}^{-} \alpha\right)^{-b_{-}(L)} E_{\mathcal{C}}(D) \alpha^{\otimes n},
$$

where $D$ is any Hopf diagram such that $L$ is isotopic to $\phi(D) \circ(\cup \otimes \cdots \otimes \cup)$.

Remarks 1) Corollary 5.5 gives an intrinsic description, in terms of Hopf algebraic structures, of quantum invariants of 3-manifolds.

2) In Corollary [5.5. we can in particular take $D=\Psi(T)$ where $\Psi$ is as in Theorem 4.5 and $T$ is a ribbon sting link such that $L$ is isotopic to closure of $T$. Recall also that the constructive proof of Theorem 4.5] provides us with an algorithm for computing $D=\Psi(T)$ starting from a diagram of $T$.

Let us conclude by giving an example. Recall that the Poincaré sphere $\mathbb{P}$ can be presented as $\mathbb{P} \simeq S_{K}^{3}$ where $K$ is the right-handed trefoil with framing +1 . Now this trefoil can be represented as $K=\phi(D) \circ \cup$ where $D=\left(\omega_{+} \Delta \otimes \theta_{-}\right) \Delta$. Indeed, we have:

$$
K=A_{\sigma b} \text { and } D=Y_{1} \rightarrow \phi(D)=S_{1}
$$

Hence, if $\alpha: \mathbf{1} \rightarrow A$ is a normalizable Kirby element of $\mathcal{C}$, then:

$$
\tau_{\mathcal{C}}(\mathbb{P} ; \alpha)=\theta_{\alpha}^{-1}
$$

\section{References}

[1] A Bruguières, Tresses et structure entière sur la catégorie des représentations de $\mathrm{SL}_{N}$ quantique, Comm. Algebra 28 (2000) 1989-2028 MathReview

[2] A Bruguières, Double Braidings, Twists, and Tangle Invariants (2004), to appear in J. Pure Appl. Alg. arXiv:math.QA/0407217

[3] T Kerler, V V Lyubashenko, Non-semisimple topological quantum field theories for 3-manifolds with corners, Lecture Notes in Mathematics 1765, SpringerVerlag, Berlin (2001) MathReview 
[4] R Kirby, A calculus for framed links in $S^{3}$, Invent. Math. 45 (1978) 35-56 MathReview

[5] W B R Lickorish, An introduction to knot theory, Graduate Texts in Mathematics 175, Springer-Verlag, New York (1997) MathReview

[6] V Lyubashenko, Modular transformations for tensor categories, J. Pure Appl. Algebra 98 (1995) 279-327 MathReview

[7] V V Lyubashenko, Invariants of 3-manifolds and projective representations of mapping class groups via quantum groups at roots of unity, Comm. Math. Phys. 172 (1995) 467-516 MathReview

[8] S Mac Lane, Categories for the working mathematician, Graduate Texts in Mathematics 5, Springer-Verlag, New York (1998) MathReview

[9] S Majid, Braided groups, J. Pure Appl. Algebra 86 (1993) 187-221 MathReview

[10] A Markoff, Foundations of the algebraic theory of tresses, Trav. Inst. Math. Stekloff 16 (1945) 53 MathReview

[11] MHA Newman, On theories with a combinatorial definition of "equivalence.", Ann. of Math. (2) 43 (1942) 223-243 MathReview

[12] N Reshetikhin, V G Turaev, Invariants of 3-manifolds via link polynomials and quantum groups, Invent. Math. 103 (1991) 547-597 MathReview

[13] V G Turaev, Quantum invariants of knots and 3-manifolds, de Gruyter Studies in Mathematics 18, Walter de Gruyter \& Co., Berlin (1994) MathReview

[14] A Virelizier, Kirby elements and quantum invariants, to appear in Proc. London Math. Soc. arXiv:math.GT/0312337

I3M, Université Montpellier II, 34095 Montpellier Cedex 5, France

and

Department of Mathematics, University of California, Berkeley CA 94720, USA

Email: bruguier@math.univ-montp2.fr and virelizi@math.berkeley.edu

Received: 13 June 2005 